\title{
Theory of Phonon-Assisted Multimagnon Optical Absorption and Bimagnon States in Quantum Antiferromagnets.
}

\author{
J. Lorenzanal and G. A. Sawatzky \\ Laboratory of Applied and Solid State Physics, Materials Science Centre, \\ University of Groningen, Nijenborgh 4, 9747 AG Groningen, The Netherlands
}

(October 10, 2018)

\begin{abstract}
We calculate the effective charge for multimagnon infrared (IR) absorption assisted by phonons in a perovskite like antiferromagnet and we compute the spectra for two magnon absorption using interacting spin-wave theory. The full set of equations for the interacting two magnon problem is presented in the random phase approximation for arbitrary total momentum of the magnon pair. The spin wave theory results fit very well the primary peak of recent measured bands in the parent insulating compounds of cuprate superconductors. The line shape is explained as being due to the absorption of one phonon plus a new quasiparticle excitation of the Heisenberg Hamiltonian that consists off a long lived virtual bound state of two magnons (bimagnon). The bimagnon states have well defined energy and momentum in a substantial portion of the Brillouin zone. The higher energy bands are explained as one phonon plus higher multimagnon absorption processes. Other possible experiments for observing bimagnons are proposed. In addition we predict the line shape for the spin one system $\mathrm{La}_{2} \mathrm{NiO}_{4}$.

78.30.Hv,75.40.Gb,75.50.Ee,74.72.-h
\end{abstract}

\section{INTRODUCTION}

In the late 50's Newman and Chrenko presented a pioneering infrared absorption study in $\mathrm{NiO}$ [1]. Among other results they showed a band at $0.24 \mathrm{eV}$ that correlated with the disappearance of antiferromagnetism above the Néel temperature and hence of likely magnetic origin. This was very puzzling because in the $\mathrm{NaCl}$ structure, with an $\mathrm{O}$ at an inversion center, a direct magnetic absorption is not allowed [2]. In fact in a typical two magnon excitation, like for example, two spin flips on adjacent metals atoms, all relaxation of charge in the excited state is symmetric with no net dipole moment. An explanation of this puzzle was given by Mizuno and Koide [3] who observed that the joint absorption process of a phonon and two magnons is allowed since then the symmetry of the lattice, after the phonon excitation, is effectively lower. To the best of our knowledge no detailed theory existed of this effect. Note that the Mizuno and Koide [3] paper is from the early days of Anderson's superexchange theory.

Also very puzzling data was recently presented by Perkins et al [4.5. They measured the absorption in many different parent insulating compounds of high- $T_{c}$ superconductors. The data shows a narrow primary peak in the charge transfer gap and a set of side bands. Initially the narrow peak was associated with an unidentified exciton. However no exciton is expected in this energy range [6,7].

The propose of this paper is to present a detailed theory of phonon-assisted multimagnon infrared absorption. We apply the theory to layered insulators and show that it explains Perkins et al. data (Sec. IV). We estimate the coupling constant of light with multimagnon excitations (Sec. III) and we calculate the line shape for one phonon plus two magnon absorption using interacting spin wave theory. For this we need to solve the two-magnon problem for arbitrary total momentum. We present the full set of equations in the random-phase-approximation (RPA) and solve them approximately (Sec. IIII). The narrow primary peak reproduced in Fig. 10 is explained in terms of a new quasiparticle excitation. It consists of a long lived virtual bound state of magnons referred to here as a bimagnon. The new state has well defined energy and momentum in a substantial portion of the Brillouin zone. We also present a prediction for the line shape of the spin one system $\mathrm{La}_{2} \mathrm{NiO}_{4}$.

So far complementary information on antiferromagnetism in perovskite materials has come from probes like neutron scattering and Raman light scattering [8]. Our results show that IR absorption can be used to study magnetic properties of this systems. This is interesting because the technique is relatively simple.

In the conclusions (Sec. V) we discuss and propose other experiments which should be able to detect the new excitations.

A short account of this results was presented elsewhere [9].

\section{THEORY OF PHONON-ASSISTED IR ABSORPTION OF MAGNONS}

\section{A. Model Hamiltonian}

We concentrate on the case of a spin $S=1 / 2$ and 2 dimensional $(2-\mathrm{d})$ material like the $\mathrm{Cu}-\mathrm{O}$ layers of the cuprates but we indicate the corresponding generalizations for a system with larger dimension and/or spin like $\mathrm{La}_{2} \mathrm{NiO}_{4}$ or $\mathrm{NiO}$. We consider a three-band-Peierls- 
Hubbard model [10,11] in the presence of an electric field $(\boldsymbol{E})$,

$$
\begin{aligned}
H= & \sum_{i \neq j, \sigma} t_{i j}\left(\left\{\boldsymbol{u}_{k}\right\}\right) c_{i \sigma}^{\dagger} c_{j \sigma}+\sum_{i, \sigma} e_{i}\left(\left\{\boldsymbol{u}_{k}\right\}, \boldsymbol{E}\right) c_{i \sigma}^{\dagger} c_{i \sigma} \\
& +\sum_{i} U_{i} c_{i \uparrow}^{\dagger} c_{i \downarrow}^{\dagger} c_{i \downarrow} c_{i \uparrow}+\sum_{\langle i \neq j\rangle, \sigma, \sigma^{\prime}} U_{i j} c_{i \sigma}^{\dagger} c_{j \sigma^{\prime}}^{\dagger} c_{j \sigma^{\prime}} c_{i \sigma} \\
& +\sum_{l} \frac{1}{2 M_{l}} \boldsymbol{P}_{l}^{2}+\sum_{k, l} \frac{1}{2} \boldsymbol{u}_{k} \mathcal{K}_{k l} \boldsymbol{u}_{l}-\boldsymbol{E} \boldsymbol{P}_{\mathrm{ph}} .
\end{aligned}
$$

Here, $c_{i \sigma}^{\dagger}$ creates a hole with spin $\sigma$ at site $i$ in the $\mathrm{Cu}$ $d_{x^{2}-y^{2}}$ or the $\mathrm{O} p_{x, y}$ orbital. For simplicity $\mathrm{Cu}$ atoms are kept fixed and $\mathrm{O}$ atoms are allowed to move with displacements $\boldsymbol{u}_{k}$. These restrictions will be effectively removed when we compare with real experiments since measured phonon properties will be used. $\mathcal{K}_{k l}$ is a spring constant tensor and $\boldsymbol{P}_{l}$ is the momentum canonically conjugate to $\boldsymbol{u}_{l}, \boldsymbol{P}_{\mathrm{ph}}=Z e \sum_{i} \boldsymbol{u}_{k}$ is the phonon dipolar moment and $Z$ is the ionic charge of $\mathrm{O}(Z=-2)$. For electron-lattice coupling, we assume that the nearestneighbor $\mathrm{Cu}-\mathrm{O}$ hopping is modified by the $\mathrm{O}$-ion displacement $\boldsymbol{u}_{k}$ as $t_{i j}=t \pm \alpha \boldsymbol{\delta} \boldsymbol{u}_{k}$, where $\boldsymbol{\delta}$ is a unit vector in the direction of the corresponding $\mathrm{Cu}-\mathrm{Cu}$ bond, $\boldsymbol{\delta}=\hat{\boldsymbol{x}}$, $\hat{\boldsymbol{y}}$ (and $\hat{\boldsymbol{z}}$ for a 3 -d system) and the $+(-)$ applies if the $\mathrm{Cu}-\mathrm{O}$ bond shrinks (stretches) with positive $\boldsymbol{\delta} \boldsymbol{u}_{k}$ (O-O hopping is neglected here). $t$ is given by $1 / 2 \sqrt{3} p d \sigma$ in terms of Slater-Koster integrals [12]. The site energy contains the coupling to the electric field and in addition the $\mathrm{Cu}$ site energy is assumed to be modulated linearly by the displacements of the $\mathrm{O}$ ions,

$$
\begin{aligned}
& e_{i}=E_{d}+\beta \sum_{k}( \pm) \boldsymbol{\delta} \boldsymbol{u}_{k}+e \boldsymbol{E} \boldsymbol{r}_{i} \quad(\mathrm{Cu}), \\
& e_{i}=E_{p}+e \boldsymbol{E} \boldsymbol{r}_{i} \quad(\mathrm{O}) .
\end{aligned}
$$

In Eq. (2) the sum extends over the four surrounding $\mathrm{O}$ ions. The sign takes the value $+(-)$ if the bond becomes longer (shorter) with positive $\boldsymbol{\delta} \boldsymbol{u}_{k}$ and $\boldsymbol{r}_{i}$ is the position of atom $i$ (including displacement). The other electronic matrix elements are: $\mathrm{Cu}$-site $\left(U_{d}\right)$ and O-site $\left(U_{p}\right)$ repulsions for $U_{i}$, and the nearest-neighbor $\mathrm{Cu}-\mathrm{O}$ repulsion $\left(U_{p d}\right)$. We define $\Delta=E_{p}-E_{d}+U_{p d}$ and $\epsilon=2\left(E_{p}-E_{d}\right)+U_{p}$. In the following we adopt the notation that $\mathrm{Cu}$ sites are labeled with $i$ and $\mathrm{O}$ sites or the corresponding $\mathrm{Cu}-\mathrm{Cu}$ bond with $\boldsymbol{i}+\boldsymbol{\delta} / 2$. In this notation the position of an $\mathrm{O}$ ion is given by $\boldsymbol{r}_{\boldsymbol{i}+\boldsymbol{\delta} / 2}=a(\boldsymbol{i}+\boldsymbol{\delta} / 2)+\boldsymbol{u}_{\boldsymbol{i}+\boldsymbol{\delta} / 2}$, with $a$ the lattice constant.

\section{B. Effective Charges and Dipole Moment Operator}

To calculate the coupling constants of light with oneand two-phonon-multimagnon processes we first obtain a low energy Hamiltonian as a perturbation expansion valid when $t<<\Delta, \epsilon, U_{d}$ and when the phonon field and the electric field vary slowly with respect to typical gap frequencies,

$$
H=\sum_{\boldsymbol{i}, \boldsymbol{\delta}} J\left(\boldsymbol{E},\left\{\boldsymbol{u}_{\boldsymbol{i}+\boldsymbol{\delta} / 2}\right\}\right) B_{\boldsymbol{i}+\boldsymbol{\delta} / 2}+H_{\mathrm{ph}}-\boldsymbol{E} \boldsymbol{P}_{\mathrm{ph}} .
$$

Here $B_{\boldsymbol{i}+\boldsymbol{\delta} / 2}=\boldsymbol{S}_{\boldsymbol{i}} \boldsymbol{S}_{\boldsymbol{i}+\boldsymbol{\delta}}$ with $\boldsymbol{S}_{\boldsymbol{i}}$ spin operators, $H_{\mathrm{ph}}$ is the phonon Hamiltonian containing spring constants and masses for the $\mathrm{O}$ ions $(M)$ and $\boldsymbol{P}_{\mathrm{ph}}$. The first term in Eq. (1) contains the spin-dependent fourth order correction in $t$ whereas fourth, second and zero order spinindependent processes are collected in the last two terms. To compute $J$ we can use the three center system $\mathrm{Cu}_{\mathrm{L}^{-}}$ $\mathrm{O}-\mathrm{Cu}_{\mathrm{R}}$ of Fig. 1 .

See Ref. [9]

FIG. 1. Schematic representation of the cluster used in the calculations. Full dots represent Cu's and open dots O's. Thick arrows represent the spin, thin short arrows represent lattice displacements and thin long arrows represent the direction of the electric field. We have represented $\mathbf{u}_{0}$ in configuration A. In general its direction is equal to the direction of the electric field.

In order to generalize our result for arbitrary spin $S$ we define the saturated ferromagnetic state

$$
|\mathrm{F}\rangle=\left|m_{\mathrm{L}}=S, S_{\mathrm{L}}=S ; m_{\mathrm{R}}=S, S_{\mathrm{R}}=S\right\rangle
$$

and the Néel state,

$$
|\mathrm{N}\rangle=\left|m_{\mathrm{L}}=S, S_{\mathrm{L}}=S ; m_{\mathrm{R}}=-S, S_{\mathrm{R}}=S\right\rangle .
$$

Here $m_{\mathrm{R}, \mathrm{L}}$ is the $z$ component and $S_{\mathrm{L}, \mathrm{R}}$ is the magnitude of the total spin of the ion. The superexchange can be calculated as,

$$
J=\frac{1}{S^{2}}(\langle\mathrm{~F}|V R V R V R V| \mathrm{F}\rangle-\langle\mathrm{N}|V R V R V R V| \mathrm{N}\rangle)
$$

where $R=(1-P) /\left(E_{0}-H_{0}\right), P$ projects on the manifold with $S_{\mathrm{L}}=S_{\mathrm{R}}=S, H_{0}$ contains all site-diagonal terms 
of the Hamiltonian Eq. (11) and $V$ the $d-p$ hybridization terms. For details see Ref. [13]. In the case of a $3-d$ $\mathrm{S}=1$ system like $\mathrm{NiO}$ we can orient the $z$ axis in the direction of the $\mathrm{Ni}-\mathrm{O}-\mathrm{Ni}$ bond, then $t$ should be taken as the hybridization between $d_{3 z^{2}-r^{2}}$ orbitals and the $p_{z}$ orbital and is given by $p d \sigma$ in terms of Slater and Koster integrals [12]. Also appropriate values of $U_{d}$ should be used 14. The same is valid for $\mathrm{La}_{2} \mathrm{NiO}_{4}$ if the crystal field splitting between the $d_{3 z^{2}-r^{2}}$ and the $d_{x^{2}-y^{2}}$ orbitals is neglected.

We only need to consider the three configurations (A,B,C) of the L-R bond and the electric field. Next we Taylor expand $J$ to first order in $\boldsymbol{E}$ and second order in $\left\{\boldsymbol{u}_{\boldsymbol{i}+\boldsymbol{\delta} / 2}\right\}$,

$$
\begin{aligned}
J & =J_{0}+\eta\left(u_{\mathrm{L}}-u_{\mathrm{R}}\right)-E\left[q_{\mathrm{I}} u_{0}+\lambda q_{\mathrm{A}}\left(2 u_{0}-u_{\mathrm{L}}-u_{\mathrm{R}}\right)\right] \\
& -E\left[\xi_{\mathrm{I}} u_{0}\left(u_{\mathrm{L}}-u_{\mathrm{R}}\right)+\lambda \xi_{A}\left(2 u_{0}-u_{\mathrm{L}}-u_{\mathrm{R}}\right)\left(u_{\mathrm{L}}-u_{\mathrm{R}}\right)\right] \\
& +\ldots
\end{aligned}
$$

Here $\lambda=1$ for configuration $\mathrm{A}$ and $\lambda=0$ for configurations $\mathrm{B}$ and $\mathrm{C}$. In each configuration the displacement of the central $\mathrm{O}$ and the electric field are parallel, i.e. $\boldsymbol{E}=E \hat{\boldsymbol{e}}, \boldsymbol{u}_{0}=u_{0} \hat{\boldsymbol{e}}$. The direction of $\hat{\boldsymbol{e}}$ is the same as the arrows at the bottom of Fig. 11. $u_{\mathrm{L}}$ and $u_{\mathrm{R}}$ are only relevant in configuration A. $u_{\mathrm{L}}=u_{\mathrm{L} 1}+u_{\mathrm{L} 2}-u_{\mathrm{L} 3}$, $u_{\mathrm{R}}=-u_{\mathrm{R} 1}+u_{\mathrm{R} 2}+u_{\mathrm{R} 3}$. The numbering and the direction of the displacements are shown in Fig. 11. Other terms quadratic in $u$ 's have been neglected, they renormalize the spring constants in $H_{\mathrm{ph}}$ and give the magnon-twophonon interaction. The first term in Eq. (6) is the superexchange in absence of the electric and phonon fields,

$$
J_{0}=\frac{t^{4}}{S^{2} \Delta^{2}}\left[\frac{1}{U_{d}}+\frac{2}{\epsilon}\right] .
$$

The remaining quantities are a magnon-phonon coupling constant,

$$
\eta=\frac{-t^{4} \beta}{S^{2} \Delta^{2}}\left[\frac{1}{\Delta}\left(\frac{1}{U_{d}}+\frac{2}{\epsilon}\right)+\frac{2}{\epsilon^{2}}\right],
$$

effective charges associated with one phonon and multimagnon processes,

$$
\begin{aligned}
q_{\mathrm{I}} & =-e \frac{2 t^{4}}{S^{2} \Delta^{2}}\left[\frac{1}{\Delta}\left(\frac{1}{U_{d}}+\frac{2}{\epsilon}\right)+\frac{2}{\epsilon^{2}}\right] \\
q_{\mathrm{A}} & =-e \frac{t^{4}}{S^{2} \Delta^{2}} \beta a_{p d}\left[\frac{2}{\Delta^{2}}\left(\frac{1}{U_{d}}+\frac{2}{\epsilon}\right)+\frac{1}{U_{d}}\left(\frac{1}{\Delta}+\frac{2}{U_{d}}\right)^{2}\right]
\end{aligned}
$$

and effective charges associated with two-phonon and multimagnon processes.

$$
\begin{aligned}
& \xi_{\mathrm{I}}=e \frac{t^{4} \beta}{S^{2} \Delta^{2}}\left[\frac{3}{\Delta^{2}}\left(\frac{1}{U_{d}}+\frac{2}{\epsilon}\right)+\frac{8}{\epsilon^{2}}\left(\frac{1}{\Delta}+\frac{1}{\epsilon}\right)^{2}\right] \\
& \xi_{\mathrm{A}}=-e \frac{2 t^{4} \beta^{2} a_{p d}}{S^{2} \Delta^{3}}\left(\frac{2}{U_{d}^{3}}+\frac{3}{\Delta U_{d}^{2}}+\frac{4}{\epsilon \Delta^{2}}+\frac{2}{\epsilon^{2} \Delta}+\frac{3}{U_{d} \Delta^{2}}\right)
\end{aligned}
$$

$a_{p d}$ is the $\mathrm{Cu}-\mathrm{O}$ distance, $a / 2$. Within a point charge estimation the parameter $\beta a_{p d} \approx 2 U_{p d}$. The dipole moment is obtained from Eq. (4) as $\boldsymbol{P}=-\frac{\partial H}{\partial \boldsymbol{E}}$ and using Eq. (6) in the relevant configurations. We get up to fourth order in $t$,

$$
\boldsymbol{P}=\boldsymbol{P}_{1 \mathrm{ph}}+\boldsymbol{P}_{2 \mathrm{ph}}+\boldsymbol{P}_{1 \mathrm{ph}+\mathrm{mag}}+\boldsymbol{P}_{2 \mathrm{ph}+\mathrm{mag}}
$$

The first two terms describe conventional one and two phonon absorption processes.

We define $\delta B_{\boldsymbol{i}+\boldsymbol{\delta} / 2}=B_{\boldsymbol{i}+\boldsymbol{\delta} / 2}-\left\langle B_{\boldsymbol{i}+\boldsymbol{\delta} / 2}\right\rangle$ and its Fourier transform,

$$
\delta B_{\boldsymbol{p}}^{\delta}=\frac{1}{N} \sum_{\boldsymbol{i}} e^{i \boldsymbol{p} \boldsymbol{i}} \delta B_{\boldsymbol{i}+\boldsymbol{\delta} / 2},
$$

The lattice spacing $a$ and $\hbar$ are set to 1 . The supraindex labels the direction of the vector $\boldsymbol{\delta}$ and $N$ is the number of unit cells. In the same way the Fourier transform of $\boldsymbol{u}_{\boldsymbol{i}+\boldsymbol{\delta} / 2}$ is given by

$$
\boldsymbol{u}_{\boldsymbol{p}}^{\delta}=\frac{1}{N} \sum_{\boldsymbol{i}} e^{i \boldsymbol{p} \boldsymbol{i}} \boldsymbol{u}_{\boldsymbol{i}+\boldsymbol{\delta} / 2} .
$$

After Fourier transforming, the dipole moment for one phonon and multimagnon processes for an in-plane field in the $x$ direction is,

$$
\begin{aligned}
& P_{1 \mathrm{ph}+\mathrm{mag}}^{x}=N\left[q_{\mathrm{I}} \sum_{\boldsymbol{p} \delta} \delta B_{-\boldsymbol{p}}^{\delta} u_{x \boldsymbol{p}}^{\delta}\right. \\
& \left.+\lambda 4 q_{\mathrm{A}} \sum_{\boldsymbol{p} \delta} \sin \left(\frac{p_{x}}{2}\right) \sin \left(\frac{p_{\delta}}{2}\right) \delta B_{-\boldsymbol{p}}^{x} u_{\delta \boldsymbol{p}}^{\delta}\right]
\end{aligned}
$$

and $\lambda=1$. For an electric field perpendicular to the plane we have,

$$
P_{1 \mathrm{ph}+\mathrm{mag}}^{z}=q_{\mathrm{I}} \sum_{\boldsymbol{p} \delta} \delta B_{-\boldsymbol{p}}^{\delta} u_{z \boldsymbol{p}}^{\delta} .
$$

In a 3 -d system like $\mathrm{NiO}, \lambda=1$ and we get the analogous of Eq. (15) for the three directions and the sum over $\delta$ is also for the three directions.

The term proportional to $q_{\mathrm{I}}$ is isotropic being present in any configuration. Looking at the cluster in Fig. 1 it can be understood as a spin dependent correction to the charge on $\mathrm{O}_{0}$. Its physical origin is that fourth order corrections to the charges involve spin dependent processes. For example if the spins in $\mathrm{Cu}_{\mathrm{L}}$ and $\mathrm{Cu}_{\mathrm{R}}$ are parallel they cannot both transfer to $\mathrm{O}_{0}$ whereas if they are antiparallel they can. Fig. 2(a) illustrates a typical process efficient in configuration B.

An alternative way of deriving $q_{\mathrm{I}}$ is with the aid of the Hellmann-Feynman theorem. We can add to the electronic Hamiltonian of the $\mathrm{Cu}_{\mathrm{L}}-\mathrm{O}-\mathrm{Cu}_{\mathrm{R}}$ a term $\varepsilon n_{\mathrm{O}}$ where $n_{\mathrm{O}}=n_{\mathrm{O} \uparrow}+n_{\mathrm{O} \downarrow}$ and $n_{\mathrm{O} \sigma}$ is the occupation number operator for the central $\mathrm{O}$ ion and spin $\sigma$. Now we can calculate the expectation values of the resulting Hamiltonian $H(\varepsilon)$ in the ground state $|\varepsilon\rangle$. We have that the 
total charge on the $\mathrm{O}$ is given by the following derivative of the energy,

$$
\left\langle 0\left|n_{\mathrm{O}}\right| 0\right\rangle=\left.e \frac{\partial\langle\varepsilon|H(\varepsilon)| \varepsilon\rangle}{\partial \varepsilon}\right|_{\varepsilon=0} .
$$

Since the term proportional to $J_{0}(\varepsilon)$ in $\langle\varepsilon|H(\varepsilon)| \varepsilon\rangle$ is the magnetic part of the energy its derivative is related to the magnetic dependent part of the $\mathrm{O}$ charge,

$$
q_{\mathrm{I}}=\left.e \frac{\partial J_{0}(\varepsilon)}{\partial \varepsilon}\right|_{\varepsilon=0},
$$

and since $\varepsilon$ just renormalizes $\Delta$ we have formally

$$
q_{\mathrm{I}}=e \frac{\partial J_{0}}{\partial \Delta}
$$

This expression is useful to obtain $q_{\mathrm{I}}$ if one can get $J_{0}$ from a different method.

See Ref. 9]

FIG. 2. Typical processes contributing to the isotropic (a) and anisotropic (b) effective charges. The meaning of the symbols is the same as in Fig. 2 .

The term proportional to $q_{\mathrm{A}}$ in Eq. (15) is anisotropic being present for an in-plane field only or when there are $\mathrm{Cu}-\mathrm{Cu}$ bonds oriented in the direction of the field. It originates from a "charged phonon" like effect 15]. Consider the configuration in which the electric field and the displacement of $\mathrm{O}_{0}$ are both parallel to the $\mathrm{Cu}_{\mathrm{L}}-\mathrm{Cu}_{\mathrm{R}}$ bond (A in Fig. 1), and a phonon in which the O's around $\mathrm{Cu}_{\mathrm{L}}$ breathe in and the O's around $\mathrm{Cu}_{\mathrm{R}}$ breathe out. (We don't need to consider zero momentum phonons to couple to light since it is the total momentum, magnons plus phonons which has to add to zero.) The Madelung potential in $\mathrm{Cu}_{\mathrm{R}}$ decreases, and in $\mathrm{Cu}_{\mathrm{L}}$ it increases, creating a displacement of charge from left to right that contributes to the dipole moment. Fig. 2(b) illustrates a typical process. But again this effect is spin dependent since if the two spins are parallel they cannot both transfer to $\mathrm{Cu}_{\mathrm{R}}$ and hence one gets a spin dependent correction to the dipole charge. In a highly covalent material like the cuprates one expect the charge phonon effects to be quite strong and dominate the effective charges (see Sec. IV A.

\section{Optical absorption}

The real part of the optical conductivity due to the processes described in the previous sections is given by the dipole-moment-dipole-moment correlation function. In Zubarev's notation [16

$$
\sigma=-\frac{2 \pi \omega}{N V_{\mathrm{Cu}}} \operatorname{Im}\left(\left\langle\left\langle P_{1 \mathrm{ph}+\mathrm{mag}} ; P_{1 \mathrm{ph}+\mathrm{mag}}\right\rangle\right\rangle\right)
$$

Here $V_{\mathrm{Cu}}$ is the volume associated with a $\mathrm{Cu}$ ion, i.e. $N V_{\mathrm{Cu}}$ is the total volume of the system per $\mathrm{Cu}-\mathrm{O}$ layer. Now we assume for simplicity that only the $u_{e p}^{\delta}$ 's with the same $\delta$ and $e$ mix. To zero order in the magnon-phonon interaction we can decouple the magnetic system from the phonon system. In this approximation the eigenstates of the system are products of phonon states times magnetic states and by writing the Lehmann representation of the Green function one can factor out all phonon matrix elements. Using that

$$
\left\langle 0_{\mathrm{ph}}\left|u_{e \boldsymbol{p}}^{\delta} u_{e-\boldsymbol{p}}^{\delta}\right| 0_{\mathrm{ph}}\right\rangle=\frac{1}{2 M \omega_{e p}^{\delta} N}
$$

with $\left|0_{\mathrm{ph}}\right\rangle$ the phonon vacuum and $\omega_{e p}^{\delta}$ the phonon frequency. We get,

$$
\begin{aligned}
& \sigma=-\frac{\pi \omega}{M V_{\mathrm{Cu}}} \sum_{\boldsymbol{p}} \operatorname{Im}\left[\frac{q_{\mathrm{I}}^{2}}{\omega_{\perp \boldsymbol{p}}}\left\langle\left\langle\delta B_{-\boldsymbol{p}}^{y} ; \delta B_{\boldsymbol{p}}^{y}\right\rangle\right\rangle^{\omega_{\perp p}}\right. \\
& +\frac{16 \lambda^{2} q_{\mathrm{A}}^{2} \sin ^{2}\left(\frac{p_{x}}{2}\right) \sin ^{2}\left(\frac{p_{y}}{2}\right)+\left(4 \lambda q_{\mathrm{A}} \sin ^{2}\left(\frac{p_{x}}{2}\right)-q_{\mathrm{I}}\right)^{2}}{\omega_{\| \boldsymbol{p}}} \\
& \left.\left\langle\left\langle\delta B_{-\boldsymbol{p}}^{x} ; \delta B_{\boldsymbol{p}}^{x}\right\rangle\right\rangle^{\omega_{\| \boldsymbol{p}}}\right] .
\end{aligned}
$$

Here $\omega_{\| p}$ is the frequency of the $u_{x \boldsymbol{p}}^{x}$ and $u_{y \boldsymbol{p}}^{y}$ phonons and $\omega_{\perp \boldsymbol{p}}$ is the frequency of the $u_{x \boldsymbol{p}}^{y}$ and $u_{y \boldsymbol{p}}^{x}$ phonons. $\omega_{\| \boldsymbol{p}}$ can be associated with the frequency of $\mathrm{Cu}-\mathrm{O}$ stretching mode phonons and $\omega_{\perp p}$ with that of $\mathrm{Cu}-\mathrm{O}$ bending mode phonons. The supraindex in the Green functions indicates that the poles should be shifted by that amount. 


\section{THE TWO MAGNON PROBLEM IN INTERACTING SPIN WAVE THEORY}

To compute the magnon-magnon Green functions we use interacting spin-wave theory [8] with a HolsteinPrimakoff transformation. On the A sublattice we put

$$
\begin{aligned}
& S_{i}^{+}=\sqrt{2 S\left(1-\frac{b_{i}^{\dagger} b_{i}}{2 S}\right) b_{i}} \\
& S_{i}^{-}=b_{i}^{\dagger} \sqrt{2 S\left(1-\frac{b_{i}^{\dagger} b_{i}}{2 S}\right)} \\
& S_{i}^{z}=S-b_{i}^{\dagger} b_{i}
\end{aligned}
$$

and on the B sublattice,

$$
\begin{aligned}
& S_{i}^{+}=b_{i}^{\dagger} \sqrt{2 S\left(1-\frac{b_{i}^{\dagger} b_{i}}{2 S}\right)} \\
& S_{i}^{-}=\sqrt{2 S\left(1-\frac{b_{i}^{\dagger} b_{i}}{2 S}\right) b_{i}} \\
& S_{i}^{z}=-S+b_{i}^{\dagger} b_{i}
\end{aligned}
$$

where $b_{\boldsymbol{i}}$ is a Boson operator.

Now the Hamiltonian can be formally expanded in powers of $1 / S$. From now on we adopt a more conventional notation and drop the 0 subindex in $J_{0}$. We define $n_{i}=b_{i}^{\dagger} b_{\boldsymbol{i}}$. The Hamiltonian reads $H=E_{\text {Néel }}+H_{0}+H_{1}$. With,

$$
\begin{aligned}
& E_{\text {Néel }}=-\frac{1}{2} J S^{2} z N \\
& H_{0}=S J z \sum_{\boldsymbol{i}} n_{\boldsymbol{i}}+S J \sum_{<\boldsymbol{i} \boldsymbol{j}>}\left(b_{\boldsymbol{i}}^{\dagger} b_{\boldsymbol{j}}^{\dagger}+h . c .\right) \\
& H_{1}=-J \sum_{<\boldsymbol{i} \boldsymbol{j}>} n_{\boldsymbol{i}} n_{\boldsymbol{j}}-\frac{J}{4} \sum_{<\boldsymbol{i} \boldsymbol{j}>}\left[b_{\boldsymbol{i}}^{\dagger} b_{\boldsymbol{j}}^{\dagger}\left(n_{\boldsymbol{i}}+n_{\boldsymbol{j}}\right)+\text { h.c. }\right]
\end{aligned}
$$

$\langle\boldsymbol{i j}>$ indicates that nearest neighbor pairs are counted once in the sum, $z$ is the coordination number, $E_{\text {Néel }}$ is the classical Néel energy, $H_{0}$ is the linear spin wave Hamiltonian and $H_{1}$ is the spin-wave-spin-wave interaction. Notice that the Hamiltonian is invariant under the exchange of the sublattices and so we don't need to distinguish between them. Accordingly we work in the nonmagnetic Brillouin zone.

The non-interacting part, $H_{0}$ is diagonalized by the Bogoliubov transformation,

$$
\begin{aligned}
& Q_{k}^{\dagger}=u_{k} b_{k}^{\dagger}-v_{k} b_{-k} \\
& Q_{k}=u_{k} b_{k}-v_{k} b_{-k}^{\dagger}
\end{aligned}
$$

where $b_{\boldsymbol{k}}$ is the Fourier transform of $b_{\boldsymbol{i}}$ and

$$
\begin{aligned}
& u_{k}=\sqrt{\frac{1+\omega_{k}}{2 \omega_{k}}} \\
& v_{k}=-\operatorname{sig}\left(\gamma_{k}\right) \sqrt{\frac{1-\omega_{k}}{2 \omega_{k}}},
\end{aligned}
$$

and

$$
\begin{aligned}
\gamma_{\boldsymbol{k}} & =\frac{2}{z} \sum_{\delta} \cos \left(k_{\delta}\right), \\
\omega_{\boldsymbol{k}} & =\sqrt{1-\gamma_{\boldsymbol{k}}^{2}} .
\end{aligned}
$$

Now we can normal order the Hamiltonian with respect to the non-interacting spin-wave ground state. This is equivalent to writing the Hamiltonian as a Hartree-Fock part plus fluctuations which we latter treat in the RPA. After normal ordering the Hamiltonian can be written as,

$$
H=E_{\mathrm{Néel}}+E_{\mathrm{SW}}+\sum_{\boldsymbol{k}} E_{\boldsymbol{k}} Q_{\boldsymbol{k}}^{\dagger} Q_{\boldsymbol{k}}+V_{\mathrm{res}},
$$

where $E_{\mathrm{SW}}=\frac{1}{4} J S N z \zeta(1+\zeta / 2 S), E_{\boldsymbol{k}}=E_{\mathrm{m}} \omega_{\boldsymbol{k}}, E_{\mathrm{m}}=$ $z S J(1+\zeta / 2 S), \zeta$ is the Oguchi correction,

$$
\zeta=1-\frac{1}{N} \sum_{k} \omega_{\boldsymbol{k}}
$$

For $z=4$ as in the layered materials, $\zeta \approx 0.158 . V_{\text {res }}=$ $V_{\text {res }}^{\|}+V_{\text {res }}^{\perp}$ contains the normal ordered product of the interacting part i.e.

$$
\begin{aligned}
& V_{\mathrm{res}}^{\|}=-J \sum_{<\boldsymbol{i} \boldsymbol{j}>}: n_{\boldsymbol{i}} n_{\boldsymbol{j}}: \\
& V_{\mathrm{res}}^{\perp}=-\frac{J}{4} \sum_{<\boldsymbol{i} \boldsymbol{j}>}:\left[b_{\boldsymbol{i}}^{\dagger} b_{\boldsymbol{j}}^{\dagger}\left(n_{\boldsymbol{i}}+n_{\boldsymbol{j}}\right)+h . c .\right]:
\end{aligned}
$$

The first term originates from the Ising part of the interaction. In the Ising limit it is easy to check that the effect of this term is to shift the energy of two nearestneighbors spin flips from the noninteracting value $4 J$ to $3 J$. In the Heisenberg limit and in the case of the Raman line shape as an example, the non interacting line shape has a peak at $4 J$ and this term shifts it to close to $3 J$. This term is the more important one to get the correct line shape. The second term is a correction to the exchange due to the kinematic interaction.

The next step is to put $V_{\text {res }}$ in terms of the spin-wave operators Eq. (26). Then we evaluate the normal order and we do the RPA approximation. This consists in this case, in keeping only those terms in $V_{\text {res }}$ which create or destroy a pair of magnons. With this we get,

$$
V_{\mathrm{res}}^{\mathrm{RPA}}=\frac{1}{2 N} \sum_{1234} \delta(1+2-3-4) \Gamma_{1234} Q_{1}^{\dagger} Q_{2}^{\dagger} Q_{3} Q_{4}
$$


$1,2, \ldots$ stands for $\boldsymbol{k}_{1}, \boldsymbol{k}_{2}, \ldots$ and the vertex are given in the Appendix A.

We define

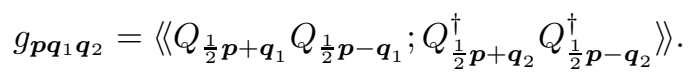

$\boldsymbol{p}$ is the total momentum of a magnon pair and $\boldsymbol{q}_{1}, \boldsymbol{q}_{2}$, the relative momentum. In the following we make indiscriminate use of the property that $g_{\boldsymbol{p} \boldsymbol{q}_{1} \boldsymbol{q}_{2}}$ does not change when $\boldsymbol{q}_{1} \rightarrow-\boldsymbol{q}_{1}$.

It is convenient for latter use to define the following auxiliary functions

$$
\begin{aligned}
& \alpha_{\boldsymbol{p} \boldsymbol{q}}^{ \pm}=u_{\frac{1}{2} \boldsymbol{p}+\boldsymbol{q}} u_{\frac{1}{2} \boldsymbol{p}-\boldsymbol{q}} \pm v_{\frac{1}{2} \boldsymbol{p}+\boldsymbol{q}} v_{\frac{1}{2} \boldsymbol{p}-\boldsymbol{q}} \\
& \beta_{\boldsymbol{p} \boldsymbol{q}}^{ \pm}=u_{\frac{1}{2} \boldsymbol{p}+\boldsymbol{q}} v_{\frac{1}{2} \boldsymbol{p}-\boldsymbol{q}} \pm v_{\frac{1}{2} \boldsymbol{p}+\boldsymbol{q}} u_{\frac{1}{2} \boldsymbol{p}-\boldsymbol{q}}
\end{aligned}
$$

the following "different sublattice" form factors,

$$
\begin{aligned}
& f_{\boldsymbol{p} \boldsymbol{q}}^{1 \delta}=\alpha_{\boldsymbol{p} \boldsymbol{q}}^{+} \cos \left(q_{\delta}\right), \\
& f_{\boldsymbol{p} \boldsymbol{q}}^{2 \delta}=\alpha_{\boldsymbol{p} \boldsymbol{q}}^{-} \cos \left(q_{\delta}\right), \\
& f_{\boldsymbol{p} \boldsymbol{q}}^{3}=\beta_{\boldsymbol{p} \boldsymbol{q}}^{+}
\end{aligned}
$$

and "same sublattice" form factors,

$$
\begin{aligned}
h_{\boldsymbol{p q}}^{1 \delta} & =\beta_{\boldsymbol{p} \boldsymbol{q}}^{+} \cos \left(q_{\delta}\right), \\
h_{\boldsymbol{p q}}^{2 \delta} & =\beta_{\boldsymbol{p} \boldsymbol{q}}^{-} \sin \left(q_{\delta}\right), \\
h_{\boldsymbol{p q}}^{3} & =\alpha_{\boldsymbol{p} \boldsymbol{q}}^{+}, \\
h_{\boldsymbol{p q}}^{4} & =\alpha_{\boldsymbol{p} \boldsymbol{q}}^{-}
\end{aligned}
$$

The $f$ 's have the property that $f_{\boldsymbol{p q}+\boldsymbol{\pi}}=-f_{\boldsymbol{p q}}$, with $\boldsymbol{\pi}=(\pi, \pi, \ldots)$. If we Fourier transform them in $\boldsymbol{q}$ we see that they are different from zero in different sublattices. For the $h$ 's, $h_{\boldsymbol{p q}+\boldsymbol{\pi}}=h_{\boldsymbol{p q}}$ and they are different from zero on the same sublattice. They will allow us to classify the Green functions in "different sublattices" Green functions and "same sublattice" Green function where the relevant coordinate is the distance between the spin operators. For example in the case of the operator Eq. (13) since $\boldsymbol{\delta}$ joints different sublattices we need the former.

By replacing $V_{\text {res }}$ by $V_{\text {res }}^{\mathrm{RPA}}$ in the Hamiltonian Eq. (30) the RPA equation of motion for the Green functions can be computed by standard methods 16 . No further approximations are needed to obtain the Eqs. (44), (45). We get

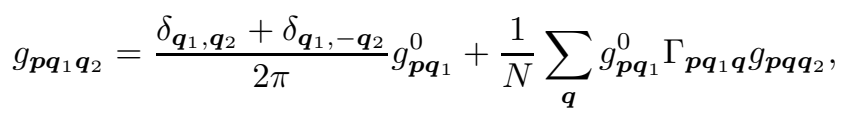

where $g_{\boldsymbol{p}, \boldsymbol{q}_{1}}^{0}=\left(\omega-E_{\frac{1}{2} \boldsymbol{p}+\boldsymbol{q}_{1}}-E_{\frac{1}{2} \boldsymbol{p}-\boldsymbol{q}_{1}}\right)^{-1}$ and the vertex is given in the Appendix A. Since the vertex is a sum of separable potentials the equations can be solved in integral form. We define same sublattice Green functions,

$$
\begin{aligned}
& K_{\mu \nu}^{(0)}=\frac{1}{N} \sum_{\boldsymbol{q}} g_{\boldsymbol{p} \boldsymbol{q}}^{0} h_{\boldsymbol{p} \boldsymbol{q}}^{\mu} h_{\boldsymbol{p} \boldsymbol{q}}^{\nu} \\
& K_{\mu \nu}=\frac{\pi}{N} \sum_{\boldsymbol{q} \boldsymbol{q}^{\prime}} g_{\boldsymbol{p} \boldsymbol{q} \boldsymbol{q}^{\prime}} h_{\boldsymbol{p} \boldsymbol{q}}^{\mu} h_{\boldsymbol{p} \boldsymbol{q}^{\prime}}^{\nu}
\end{aligned}
$$

where $\mu, \nu=l, \delta$ for $l=1,2$ and $\mu, \nu=l$ for $l=3,4$. Analogously the different sublattice Green functions are given by,

$$
\begin{aligned}
G_{\mu \nu}^{(0)} & =\frac{1}{N} \sum_{\boldsymbol{q}} g_{\boldsymbol{p} \boldsymbol{q}}^{0} f_{\boldsymbol{p} \boldsymbol{q}}^{\mu} f_{\boldsymbol{p} \boldsymbol{q}}^{\nu} \\
G_{\mu \nu} & =\frac{\pi}{N} \sum_{\boldsymbol{q} \boldsymbol{q}^{\prime}} g_{\boldsymbol{p} \boldsymbol{q} \boldsymbol{q}^{\prime}} f_{\boldsymbol{p} \boldsymbol{q}}^{\mu} f_{\boldsymbol{p} \boldsymbol{q}^{\prime}}^{\nu}
\end{aligned}
$$

It is easy to see that similar zero order Green functions with an $f$ and an $h$ factor in the kernel vanish. Since the vertex [Eq. A3)] do not mix same sublattice with different sublattice Green functions the equations for the interacting Green functions separate in two blocks. From Eq. (39) we get the following for same sublattice Green functions,

$$
\begin{aligned}
K_{\mu \nu}=K_{\mu \nu}^{(0)}-J & \left\{\sum_{\delta}\left[K_{\mu 1 \delta}^{(0)}+\frac{1}{2} \cos \left(\frac{p_{\delta}}{2}\right) K_{\mu 3}^{(0)}\right] K_{1 \delta \nu}\right. \\
& +\sum_{\delta}\left[K_{\mu 2 \delta}^{(0)}+\frac{1}{2} \sin \left(\frac{p_{\delta}}{2}\right) K_{\mu 4}^{(0)}\right] K_{2 \delta \nu} \\
& +\frac{1}{2}\left[\sum_{\delta} \cos \left(\frac{p_{\delta}}{2}\right) K_{\mu 1 \delta}^{(0)}\right] K_{3 \nu} \\
& \left.+\frac{1}{2}\left[\sum_{\delta} \sin \left(\frac{p_{\delta}}{2}\right) K_{\mu 2 \delta}^{(0)}\right] K_{4 \nu}\right\}
\end{aligned}
$$

and for different sublattices,

$$
\begin{aligned}
G_{\mu \nu}=G_{\mu \nu}^{(0)} & -J\left\{\sum_{\delta}\left[G_{\mu 1 \delta}^{(0)}+\cos \left(\frac{p_{\delta}}{2}\right) G_{\mu 3}^{(0)}\right] G_{1 \delta \nu}\right. \\
& +\sum_{\delta} G_{\mu 2 \delta}^{(0)} G_{2 \delta \nu} \\
& \left.+\left[\frac{z}{2} \gamma_{\boldsymbol{p}} G_{\mu 3}^{(0)}+\frac{1}{2} \sum_{\delta} \cos \left(\frac{p_{\delta}}{2}\right) G_{\mu 1 \delta}^{(0)}\right] G_{3 \nu}\right\}
\end{aligned}
$$

Note that the Green functions depend on the total momentum $\boldsymbol{p}$ which appears also as a parameter in the equations.

We can expand also our two magnon Green function in Eq. (21) in powers of $1 / S$ to lowest order we get,

$$
\begin{aligned}
& \left\langle\left\langle\delta B_{-\boldsymbol{p}}^{x} ; \delta B_{\boldsymbol{p}}^{x}\right\rangle\right\rangle=\frac{S^{2}}{N \pi} \times \\
& {\left[G_{1 x 1 x}+\cos \left(\frac{p_{x}}{2}\right)\left(G_{31 x}+G_{1 x 3}\right)+\cos ^{2}\left(\frac{p_{x}}{2}\right) G_{33}\right]}
\end{aligned}
$$

This neglects small Oguchi type corrections to the prefactor [17] which slightly renormalizes the intensity scales in the results that follow. First we will solve the equations in some limiting case in which one can handle the equations analytically. 


\section{A. 2-d Raman}

It is useful to see how some well known results are recovered in this formalism. In Appendix $\mathrm{B}$ we solve the equations for the case $p_{x}=p_{y}$. The case of $\boldsymbol{p}=0$ is of particular interest because it corresponds to the Raman line shape. According to Ref. [18] for the case of a layered antiferromagnet the scattered intensity is proportional to the imaginary part of the following Green function,

$$
\frac{S^{2}}{\pi} G_{\mathrm{R}} \equiv N\left\langle\left\langle B_{0}^{x}-B_{0}^{y} ; B_{0}^{x}-B_{0}^{y}\right\rangle\right\rangle=\frac{S^{2}}{\pi} G_{11, p=0}^{d} .
$$

$G_{l l^{\prime}}^{d}$ is defined in the Appendix $\mathrm{B}$ and, in this case $G_{11, \boldsymbol{p}=0}^{d(0)}=L^{(2)}, G_{22, \boldsymbol{p}=0}^{d(0)}=L^{(0)}$ and $G_{12, \boldsymbol{p}=0}^{d(0)}=L^{(1)}$ where,

$$
L^{(l)}=\frac{1}{N} \sum_{\boldsymbol{q}} \frac{f_{\boldsymbol{q}}^{2}}{\left(\omega_{\boldsymbol{q}}\right)^{l}} \frac{1}{\omega-2 E_{\boldsymbol{q}}} .
$$

and $f_{\boldsymbol{q}}=\cos q_{x}-\cos q_{y}$. With this equivalences Eq. (B2) becomes equivalent to the well known expression [17],

$$
G_{\mathrm{R}}=\frac{L^{(2)}+\frac{J}{2}\left[L^{(0)} L^{(2)}-\left(L^{(1)}\right)^{2}\right]}{1+\frac{J}{2}\left(L^{(0)}+L^{(2)}\right)+\frac{J^{2}}{4}\left[L^{(0)} L^{(2)}-\left(L^{(1)}\right)^{2}\right]} .
$$

Note that the fact that we use a Holstein-Primakoff representation rather than a Dyson-Maleev one (as in Ref. 171) did not affect this result. This is because the effect of the exchange part of the interaction which is different in the two formalisms cancels out in this case due to symmetry. In Fig. 3 we show with a solid line the imaginary part of $G_{\mathrm{R}}$ which gives the Raman line shape. The position of the maximum is at

$$
E_{(0,0)}^{\max }=1.46 E_{\mathrm{m}}=3.38 \mathrm{~J}
$$

as is well know 17]. Since the line shape lies at high energies $\left(\omega>E_{\mathrm{m}}\right)$ we can get a good approximation for $G_{11}^{d(0)}$ [Eq. (B2)] by applying the HE approximation of Appendix D. We have $G_{++}^{d(0)} \simeq G_{22}^{d(0)} \simeq G_{12}^{d(0)} \simeq G_{11}^{d(0)}$, where in this case, $G_{++, \boldsymbol{p}=0}^{d(0)}=\frac{1}{4}\left(L^{(0)}+2 L^{(1)}+L^{(2)}\right)$ and we get,

$$
G_{\mathrm{R}}=\frac{G_{++, \boldsymbol{p}=0}^{d(0)}}{1+J G_{++, \boldsymbol{p}=0}^{d(0)}} .
$$

Here the distinction mention in Appendix 1 between the HE approximation done in the vertex alone or in the vertex and in the operator does not apply because the operator has been explicitly constrained to have the correct symmetry. The corresponding line shape is given by the short dashed line in Fig. 3 for the Raman case. The position of the maximum is at $1.46 E_{\mathrm{m}}$ as before and only the intensity decrease a bit. A popular and more drastic approximation consists in putting $u_{k}=1, v_{k}=0$. In this case one gets for the Raman case,

$$
G_{\mathrm{R}}=\frac{L^{(0)}}{1+J L^{(0)}} .
$$

This line shape is shown with the long dashed line. The peak shifts appreciably and is at $1.48 E_{\mathrm{m}}$. This approximation is much worse and we will not use it in the following.

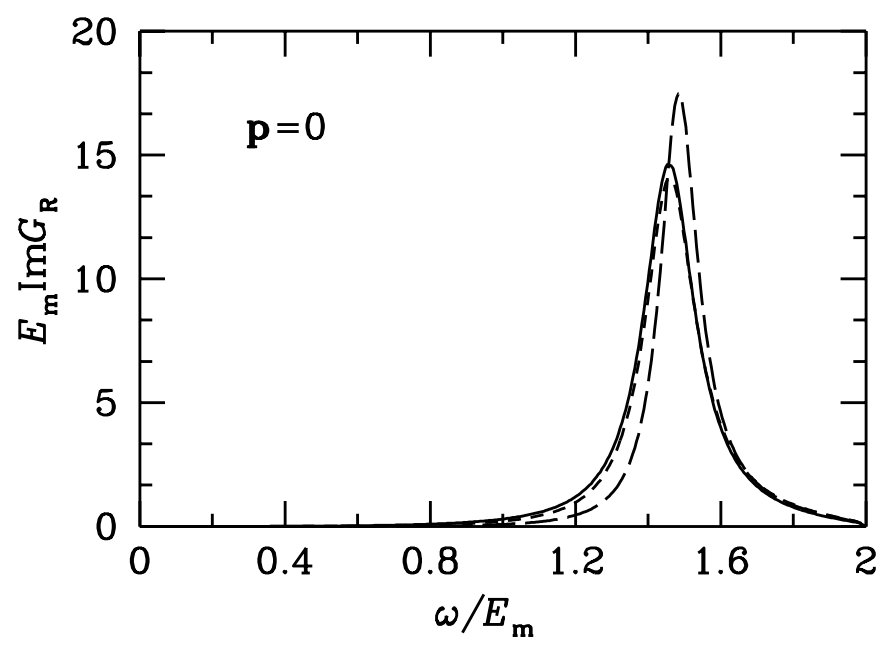

FIG. 3. Raman line shape as given by $\operatorname{Im} G_{\mathrm{R}}$ according to Eq. (49) (solid line), in the high energy (HE) approximation (short dashed line) and taking $u_{k}=1, v_{k}=0$ (long dashed line).

It is interesting to compare the present approach with the pioneering work of Elliott and Thorpe [19,20. They assume a Néel state as starting point and neglect the Oguchi correction. This gives in this case 18 a similar line shape but peaking at $2.71 \mathrm{~J}$. The present theory assumes a spin-wave ground state and takes the Oguchi correction into account. Although, at least qualitatively a similar result is obtain for $\boldsymbol{p}=0$ in the two theories, this changes dramatically for $\boldsymbol{p} \neq 0$. If one tries to extend the Elliott and Thorpe theory for that case, nonphysical poles appear in the Green functions at energies $E_{\frac{1}{2} \boldsymbol{p}+\boldsymbol{q}_{1}}-$ $E_{\frac{1}{2} \boldsymbol{p}-\boldsymbol{q}_{1}}$ indicating that a better ground state must be used as done here.

\section{B. IR case}

To calculate the IR line shape we need to evaluate the Green function given by Eq. (46). The narrow experimental line shape for the cuprates suggests the occurrence of a sharp resonance for some values of $\boldsymbol{p}$. This can not occur close to $\boldsymbol{p}=(0,0)$ or $(\pi, \pi)$ because there the continua of two magnon excitations extends from zero to $2 E_{\mathrm{m}}$ and the imaginary part of the non interacting 
Green function is relatively large at the energies of typical two-magnon excitations. This changes at different values of $\boldsymbol{p}$, in particular for $\boldsymbol{p}=(\pi, 0)$ there is a gap in the spectrum from 0 to $E_{\mathrm{m}}$ and the imaginary part of the non interacting Green function is very small where the resonance occurs. This case is analyzed in detail next.

\section{Bimagnon at $\boldsymbol{p}=(\pi, 0)$}

The relevant Green function for this problem is [Eq. (46)]

$$
\left\langle\left\langle\delta B_{-(\pi, 0)}^{x} ; \delta B_{(\pi, 0)}^{x}\right\rangle\right\rangle=\frac{S^{2}}{N \pi} G_{1 x 1 x} .
$$

In the Appendix $\mathrm{C}$ we solve Eq. (45) for this momentum in any dimension. $G_{1 x 1 x}$ is given by Eq. C2. In Fig. 4 we show with a solid line the imaginary part of this Green function. A sharp resonance occurs indicating that a virtual bound state (bimagnon) is formed. The maximum is at

$$
E_{(\pi, 0)}^{\max }=1.179 E_{\mathrm{m}}=2.731 J
$$

We show also $\operatorname{Im} G_{1 x 1 x}^{(0)}, \operatorname{Im} G_{1 x 2 x}^{(0)}, \operatorname{Im} G_{2 x 2 x}^{(0)}$ This represents the density of states of the continua of two magnon excitations in which the bimagnon can decay. As mention before it starts at $E_{\mathrm{m}}$ and is very small at the position of the bimagnon pole. This explains the long lifetime of the bimagnon. We see that $G_{1 x 1 x}^{d(0)} \simeq G_{2 x 2 x}^{d(0)} \simeq G_{1 x 2 x}^{d(0)}$.

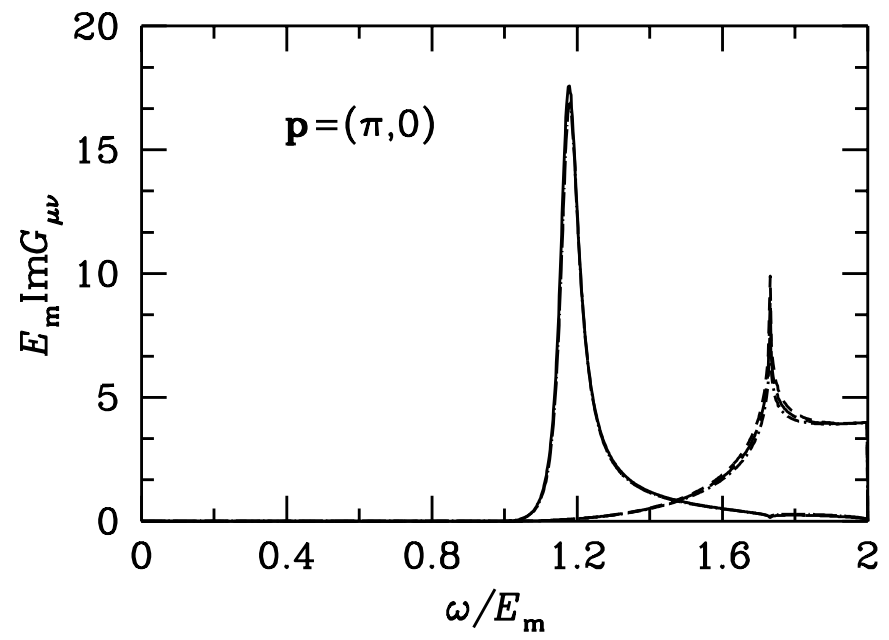

FIG. 4. $\operatorname{Im} G_{1 x 1 x}$ at $\boldsymbol{p}=(\pi, 0)$ in the full theory of Eq. C2 $)$ (solid line), and almost indistinguishable, in the HE approximation (doted long dashed line). We also show (very close to each other) $\operatorname{Im} G_{1 x 1 x}^{(0)}$ (short dashed line), $\operatorname{Im} G_{1 x 2 x}^{(0)}$ (long dashed line), $\operatorname{Im} G_{2 x 2 x}^{(0)}$ (doted short dashed line).
This is because at high energies $v_{\boldsymbol{k}}$ is quite small [see Eqs. (36a), (37), 42)] and all of them are very close to $G_{+x+x}^{(0)}$ defined in Appendix. D. We can then apply the HE approximation of Appendix. $D$. For simplicity here we use the HE approximation both in the vertex and in the operator since in this case there is no low energy spurious spectral weight i.e. we neglect $G_{+x-x}^{\prime}, G_{-x-x}^{\prime}$ in Eq. (D6a). We get the familiar RPA like form,

$$
G_{1 x 1 x}^{\prime}=\frac{G_{+x+x}^{(0)}}{1+2 J G_{+x+x}^{(0)}} .
$$

The imaginary part of this is shown with doted long dashed line in Fig. 4. Again we see that the HE approximation is very accurate. In particular the peak does not shift and there is only a small decrease in the intensity.

\section{Bimagnon for general $p$}

To obtain the total line shape we have to integrate the contributions from the whole Brillouin zone so we need the Green function at all values of $\boldsymbol{p}$. By the same arguments as before we can use the HE approximation of Appendix D. We expect this to work well because of the following reasons. i) We expect that the total line shape will be determined by the sharp excitations that occur close to momentum $(\pi, 0)$ and we have seen that in this region the approximation does extremely good. ii) Far from this region the approximation is also good as we have already shown for the Raman case.

To analyze the following results it is useful to look first at the non-interacting $(S=\infty)$ case (Fig. 5). The regions where the imaginary part is small are good candidates for having narrow resonances in the interacting case.

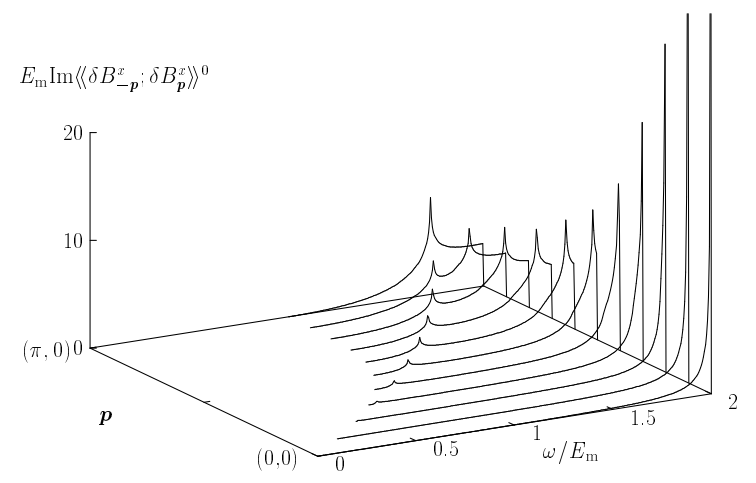



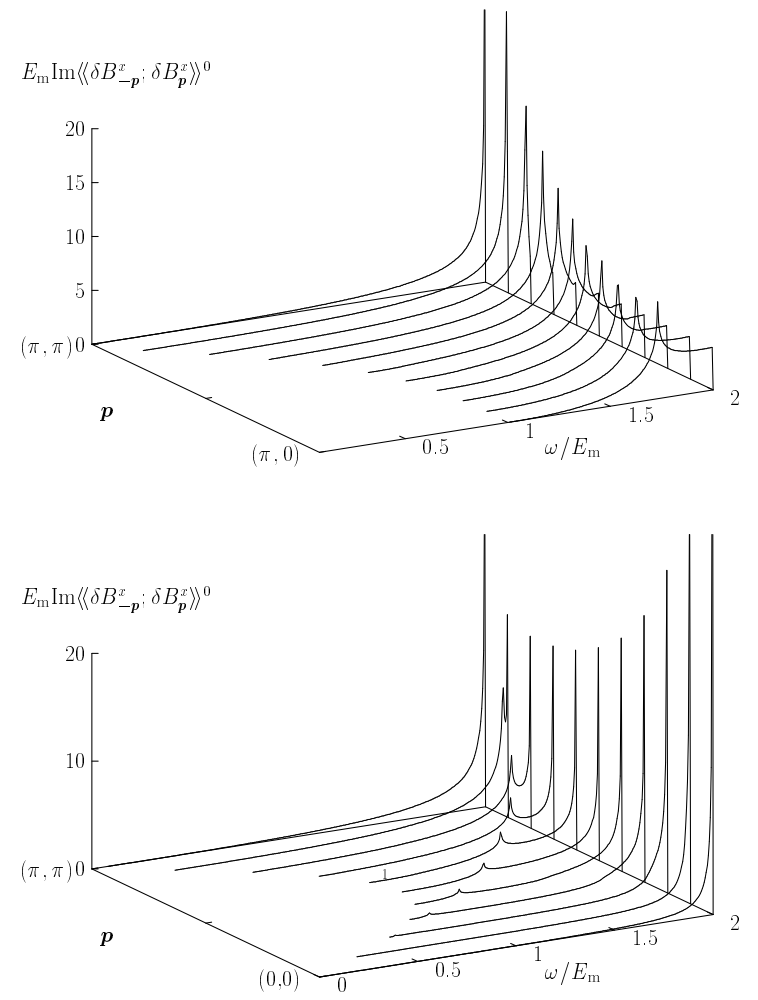

FIG. 5. $\operatorname{Im}\left\langle\left\langle\delta B_{-\boldsymbol{p}}^{x} ; \delta B_{p}^{x}\right\rangle\right\rangle$ in the noninteracting $(S=\infty)$ case as a function of $\boldsymbol{p}$, from $(0,0)$ to $(\pi, 0)$ (a); from $(\pi, 0)$ to $(\pi, \pi)(\mathrm{b})$; and from $(0,0)$ to $(\pi, \pi)(\mathrm{c})$. The regions in which the line has not been draw have zero imaginary part.

In Fig. 6 we show the imaginary part of the Green function from Eq. (46) as a function of the total momentum $\boldsymbol{p}$ in the high energy approximation in the vertex but not in the operator (see Appendix D). The bimagnon is only well defined close to $(\pi, 0)$. It disperses upwards on going towards $(0,0)$ [Fig. $6(\mathrm{a})$ ] and downwards on going towards $(\pi, \pi)$ [Fig. $6(\mathrm{~b})$ ]. This is shown more clearly in the inset of Fig. 10. This indicates that $(\pi, 0)$ is a saddle point and hence it should give a Van Hove singularity when integrated over $\boldsymbol{p}$ [Eq. (21)]. Because of that the position of the peak in the final integrated line shape is the same as for the $(\pi, 0)$ bimagnon.

The peak at lower energy is a real bound state. Its position, the intensity, and even the existence are not very reliable because the position is beyond the range of applicability of the HE approximation. If the HE approximation is done in the vertex and in the operator this peak gets a much larger spectral weight and contributes spurious intensity to the line shape (see next Section).
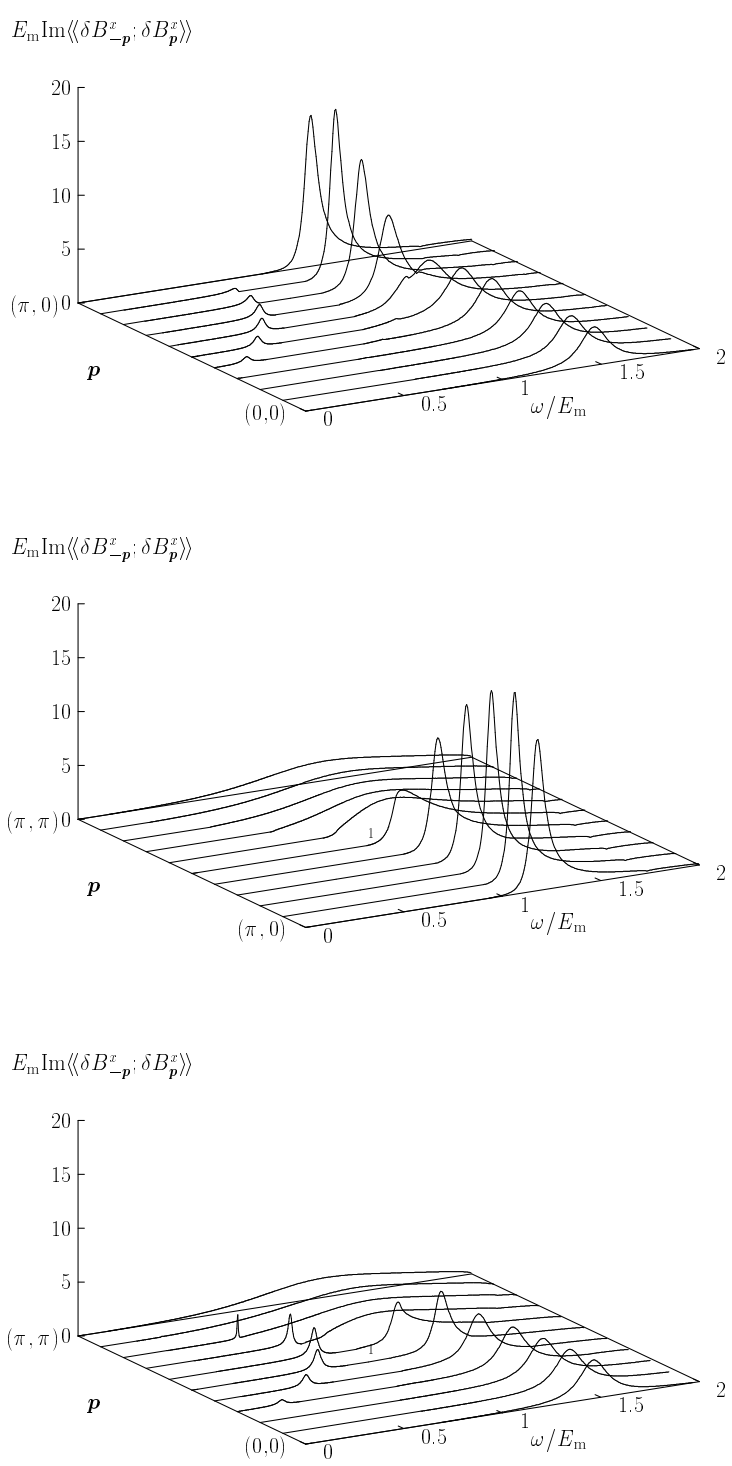

FIG. 6. $\operatorname{Im}\left\langle\left\langle\delta B_{-\boldsymbol{p}}^{x} ; \delta B_{\boldsymbol{p}}^{x}\right\rangle\right\rangle$ for $S=1 / 2$ in the HE approximation in the vertex as a function of $\boldsymbol{p}$, from $(0,0)$ to $(\pi, 0)(\mathrm{a})$; from $(\pi, 0)$ to $(\pi, \pi)(\mathrm{b})$; and from $(0,0)$ to $(\pi, \pi)(\mathrm{c})$. In the case of real bound states a small imaginary part $\left(0.02 E_{\mathrm{m}}\right)$ has been added in the denominator of the Green function.

In Fig. 7 we show the imaginary part of the Green function as a function of the total momentum $\boldsymbol{p}$ for the case of $S=1$. This will correspond to a material like $\mathrm{La}_{2} \mathrm{NiO}_{4}$. The main bimagnon peak gets overdamped in this case because the pole shifts up to the region of larger imaginary part for the noninteracting Green function (Fig. 5). 


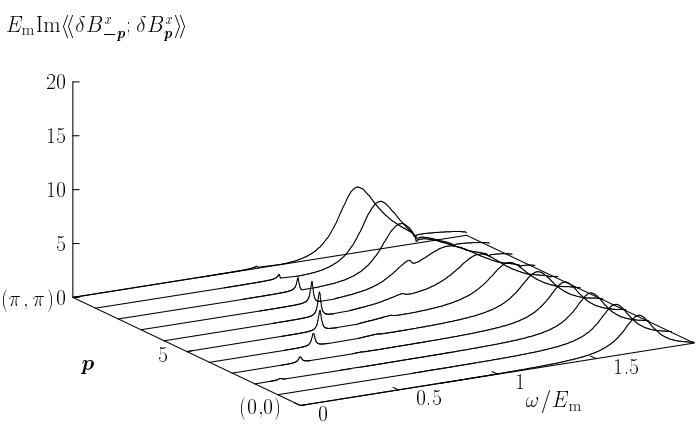

$E_{\mathrm{m}} \operatorname{Im}\left\langle\left\langle\delta B_{-p}^{x} ; \delta B_{p}^{x}\right\rangle\right\rangle$

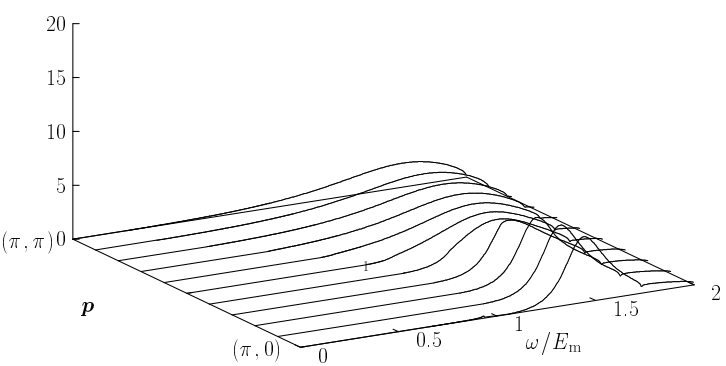

$E_{\mathrm{m}} \operatorname{Im}\left\langle\left\langle\delta B_{-p}^{x} ; \delta B_{p}^{x}\right\rangle\right\rangle$

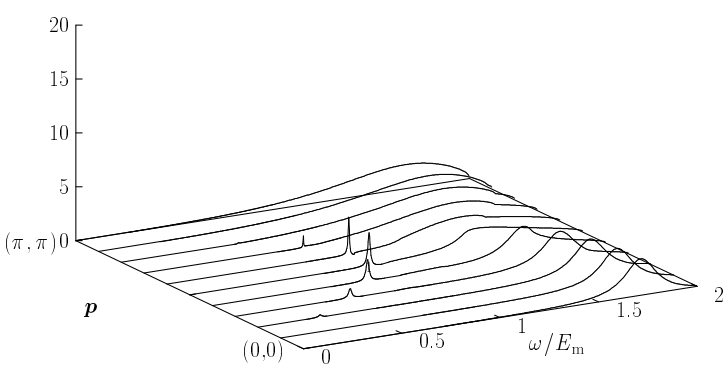

FIG. 7. $\operatorname{Im}\left\langle\left\langle\delta B_{-\boldsymbol{p}}^{x} ; \delta B_{\boldsymbol{p}}^{x}\right\rangle\right\rangle$ for $S=1$ in the HE approximation in the vertex as a function of $\boldsymbol{p}$, from $(0,0)$ to $(\pi, 0)(\mathrm{a})$; from $(\pi, 0)$ to $(\pi, \pi)(\mathrm{b})$; and from $(0,0)$ to $(\pi, \pi)(\mathrm{c})$. In the case of real bound states a small imaginary part $\left(0.02 E_{\mathrm{m}}\right)$ has been added in the denominator of the Green function.

\section{COMPARISON WITH EXPERIMENTS}

\section{A. Anisotropy}

We first discuss the anisotropy because this helps to fix the values of the effective charges. For an electric field polarized perpendicular to the plane only phonons perpendicular to the $\mathrm{Cu}-\mathrm{O}$ bond contribute. A quick estimate indicates that the absorption in this direction is roughly a factor of 8 smaller than the in-plane contribution. From the experimental side the anisotropy seems to be larger. One should be aware that the cuprates are in a regime where covalency is not small with respect to typical gap energies and hence a perturbation in $t$ is helpful to identify the important processes and discuss trends, but quantitative estimations are to be taken with care. The same problem has been pointed out for the computation of $J$ [13]. As higher orders in $t$ are included we expect that the anisotropic contributions grow with respect to the isotropic ones. For example the charged phonon effects of Fig. 2(b) can become very efficient if the second hole forms a Zhang-Rice singlet with the hole already present in $\mathrm{Cu}_{\mathrm{L}}$ since that process involves a much smaller gap. Note also that the larger the order in $t$ the longer the range of the processes that contribute to the anisotropic charges whereas only local processes contribute to the isotropic charge. Longer range processes have in general very large form factors. These effects should give a stronger anisotropy in accordance with the experiments. This suggest that the process involving $\omega_{\|}$ are dominant. We enforce that in the following by taking $q_{\mathrm{I}}=0$.

\section{B. IR line shape}

The line shape for phonon-assisted multimagnon absorption is given by Eq. (21). Since the dispersion of the phonons is very small [21] with respect to the line width we can neglect it and take Einstein phonons. It is convenient to define the following function

$$
\begin{aligned}
& I(\omega>0)=-16 \times \\
& \sum_{\boldsymbol{p}} \sin ^{2}\left(\frac{p_{x}}{2}\right)\left(\sin ^{2}\left(\frac{p_{y}}{2}\right)+\sin ^{2}\left(\frac{p_{x}}{2}\right)\right) \operatorname{Im}\left\langle\left\langle\delta B_{-\boldsymbol{p}}^{x} ; \delta B_{\boldsymbol{p}}^{x}\right\rangle\right\rangle
\end{aligned}
$$

and $I(\omega<0)=0$. Although the line shape is different for each cuprate $I(\omega)$ is the same. The line shape is given by

$$
\sigma=\sigma_{0} \omega I\left(\omega-\omega_{\|}\right)
$$

where

$$
\sigma_{0}=\frac{\pi q_{\mathrm{A}}^{2}}{M V_{\mathrm{Cu}} \omega_{\|}}
$$

The absorption coefficient is obtained assuming weak absorption as

$$
\alpha=\frac{4 \pi}{c \sqrt{\epsilon_{1}}} \sigma,
$$

with $\epsilon_{1}$ the real part of the dielectric constant [5]. We also define 


$$
\alpha_{0}=\frac{4 \pi}{c \sqrt{\epsilon_{1}}} \sigma_{0} .
$$

In Fig. 8 we show the function $I(\omega)$ for $S=1 / 2$ in the $\mathrm{HE}$ approximation, done in the vertex alone and in both the vertex and the operator. We see that at HE the two give very similar results but at lower energy the latter have a much larger feature coming from the real bound states. Most of this spectral weight is spurious and can be easily eliminated just by neglecting the contributions from the real bound states as was done in Ref. [9]. For comparison we show $I(\omega)$ for other values of $S$.

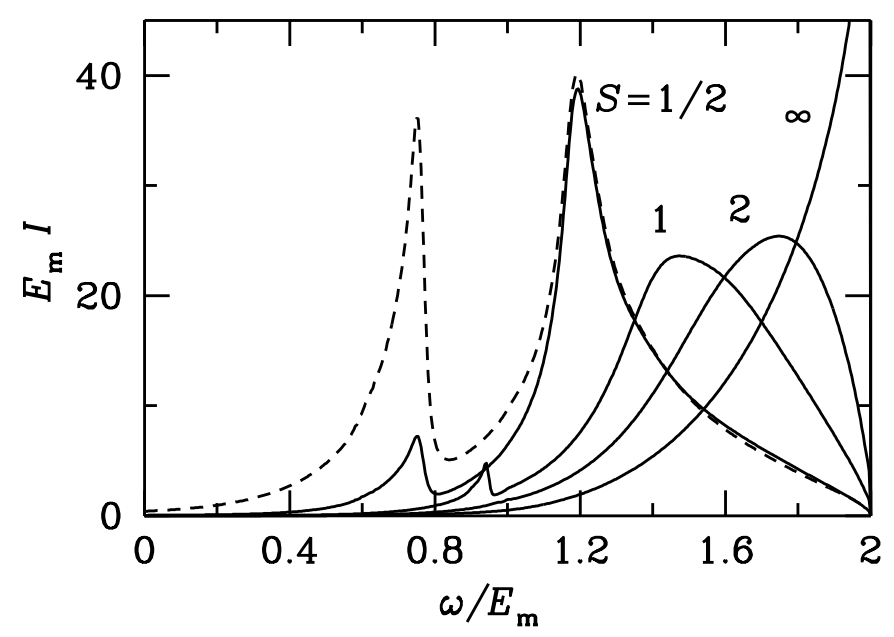

FIG. 8. $I(\omega)$ for different values of $S$ in the HE approximation done in the vertex alone (full line). In the case of $S=1 / 2$ we show also the result for the HE approximation done in the vertex and in the operator (dashed line). A small imaginary part $\left(0.016 E_{\mathrm{m}}\right)$ has been added in the denominator of the Green function.

To choose $\omega_{\|}$notice that because of the Van Hove singularity the position of the maximum in the line shape is determined by the position of the bimagnon peak at $(\pi, 0)$ [Eq. (54)] and is given by $\omega_{\|(\pi, 0)}+E_{(\pi, 0)}^{\max }$. Because of that, to optimize the position of the maximum, it is convenient to fix the Einstein phonon frequency at $\omega_{\|}=\omega_{\|(\pi, 0)}$.

The dashed curve in Fig. 9 shows the theoretical and experimental line shape in the $\mathrm{HE}$ approximation (vertex only) for $\mathrm{Sr}_{2} \mathrm{CuO}_{2} \mathrm{Cl}_{2}$. Here $\omega_{\|(\pi, 0)}$ has not been measured so we took it as $5 \%$ smaller than the measured IR frequency [22] of the $\mathrm{E}_{u}$ stretching mode as suggesting by comparing with $\mathrm{La}_{2} \mathrm{CuO}_{4}$. Notice that these differences are insignificant anyway. The fitting for the primary peak is quite good. The improved theory (HE approximation in the vertex alone) has a slightly narrower line shape which makes the fit not as good as for the more approximate line shape of Ref. [9]. Still the fit is quite good and this is surprising because such a good fit, especially for the width was not possible within RPA in the Raman case [23]. In fact the experimental Raman line shape is much broader than the theoretical prediction shown in Fig. 3. This suggest that RPA is not so accurate in the case of $S=1 / 2$. The fact that we obtain a reasonable fit in our case can be partially reconciled with the relatively bad performance of RPA in the Raman case by the fact that a structure that is artificially broadened around $p=(\pi, 0)$ by a factor of 2 (but still much narrower than the integrated line shape) does not change significantly the final result. i.e. the final width in the line shape is not so sensitive to the width of the bimagnon close to $p=(\pi, 0)$ so this experiment does not prove or disprove the accuracy of RPA for a particular momentum.

From the position of the maximum we found $J=$ $0.107 \mathrm{eV}$. An alternative way to estimate $J$ is from the position of the maximum in the Raman peak, Eq. (50). From the measurements of Ref. 24 we found $J=$ $0.103 \mathrm{eV}$. In this estimation one should again be aware that the accuracy of RPA for a $S=1 / 2$ system has been questioned 23].

We keep for comparison in Fig's. 9.10 the contribution from the real bound states although their position, intensity and even their existence are not very reliable because they are beyond the applicability of the HE approximation.

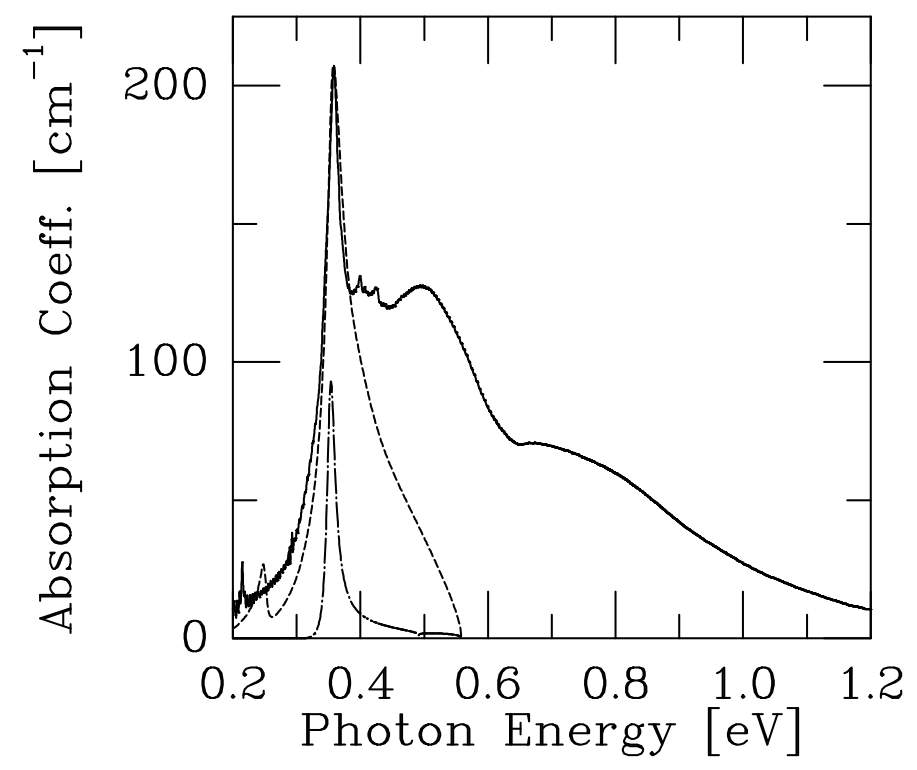

FIG. 9. Experimental data from Ref. [⿰亻⿻乚丨凵] (solid line) and theoretical line shape for two-magnon absorption (dashed line) in $\mathrm{Sr}_{2} \mathrm{CuO}_{2} \mathrm{Cl}_{2}$. The dashed doted line is the contribution to the line shape from the bimagnon at $\boldsymbol{p}=(\pi, 0)$. Parameters are $\omega_{\|}=0.061 \mathrm{eV} J=0.107 \mathrm{eV}, \alpha_{0}=3.72 \mathrm{~cm}^{-1}$

The data shows also tiny structures above the primary peck. These structures can also be due to adsorbates 25] 
or, as the same authors suggest, they can be phonon side bands. In fact the two-phonon plus bimagnon contribution is expected at $E_{(\pi, 0)}^{\max }$ plus two phonon frequencies. Its spectral weight is much smaller than the one-phonon plus bimagnon. For example the isotropic charge is of order $\xi_{\mathrm{I}} \sim \frac{\beta}{\Delta} q_{\mathrm{I}}$ and hence the absorption is of order $\left\langle\left\langle P_{2 \mathrm{ph}+\mathrm{mag}} ; P_{2 \mathrm{ph}+\mathrm{mag}}\right\rangle\right\rangle \frac{\left\langle u_{i+\delta / 2}^{2}\right\rangle}{a_{p d}^{2}}\left\langle\left\langle P_{1 \mathrm{ph}+\mathrm{mag}} ; P_{1 \mathrm{ph}+\mathrm{mag}}\right\rangle\right\rangle$, where we have taken a factor $\frac{\beta^{2} a_{p d}^{2}}{\Delta^{2}} \sim 1$. This is roughly three orders of magnitude smaller than the previous contributions and can explain the tiny structures reported for $\mathrm{Sr}_{2} \mathrm{CuO}_{2} \mathrm{Cl}_{2}$. The shift from the primary peak is $\sim$ $0.04 \mathrm{eV}$ and $0.07 \mathrm{eV}$ in good agreement with typical values of $\omega_{\perp}$ and $\omega_{\|}$. Note that only the presence of a sharp bimagnon state would make those processes observable.

In Fig. 10 we show the fit for $\mathrm{La}_{2} \mathrm{CuO}_{4}$. Here we found $J_{0}=0.121 \mathrm{eV}$. The corresponding value from the position of the maximum in the observed Raman line shape [24,23] is $J=0.118 \mathrm{eV}$. The same comment as before applies for the accuracy of this RPA estimates. The superexchange was estimated also from a study of the moments of the Raman line shape [23] with the result $J=0.128 \mathrm{eV}$.

The $\omega_{\|}$phonon is very anomalous in orthorombic $\mathrm{La}_{2} \mathrm{CuO}_{4}$ since it splits due to anharmonicities [21], its partner being at $\omega_{\|}^{\prime}=0.06 \mathrm{eV}$ at room temperature. Presumably this produces the shoulder observed at lower energies in the experiments (Fig. 10), although the distance to the primary is larger than expected which may be because other phonons are involved. This feature was assigned to direct two-magnon absorption [4] made weakly allowed by the lower lattice symmetry according to the results of Ref. [2]. However we found that the dipole moment for this process is directed in the direction bisecting an angle made by the $\mathrm{Cu}-\mathrm{O}-\mathrm{Cu}$ bond [26] and hence can only contribute for a field perpendicular to the plane. It is possible that a soft mode of the distorted structure gives rise to this effect since this will generate an enhanced intensity at lower frequencies according to Eq. (21). although more theoretical and experimental work is needed to clarify this point. Note that the shift between the primary peak and the shoulder is in the range of phonon energies $(\sim 0.04 \mathrm{eV})$.

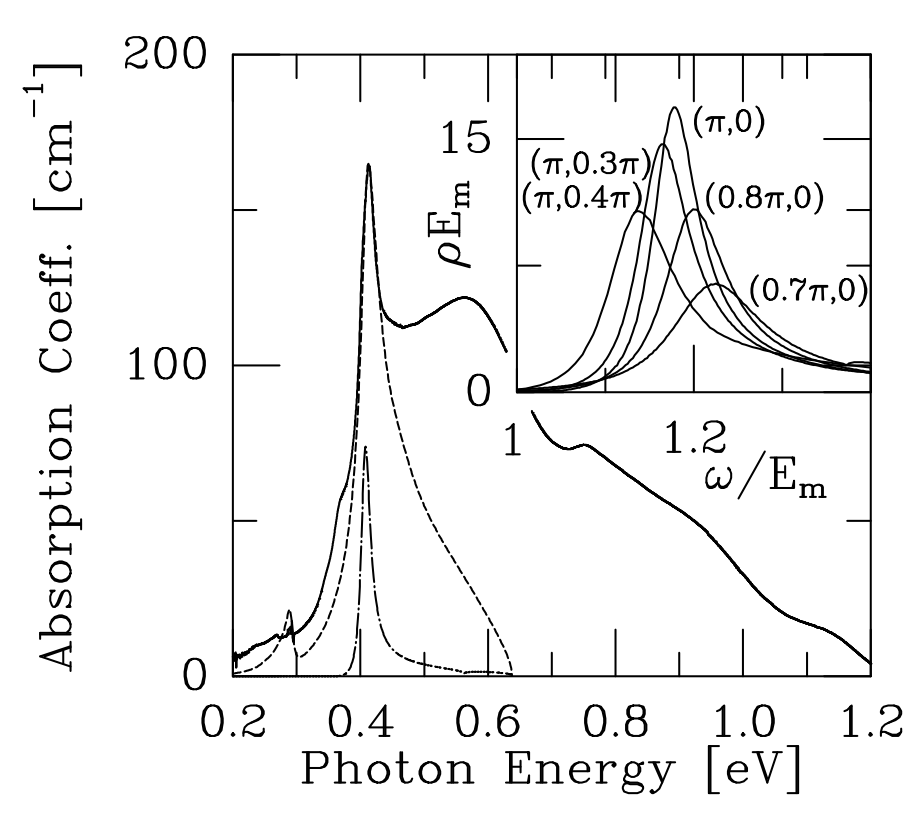

FIG. 10. Experimental data from Ref. [4] (solid line) and theoretical line shape for two-magnon absorption (dashed line) in $\mathrm{La}_{2} \mathrm{CuO}_{4}$. The dashed doted line is the contribution to the line shape from the bimagnon at $\boldsymbol{p}=(\pi, 0)$ Parameters are $\omega_{\|}=0.080 \mathrm{eV}$ [21], $J=0.121 \mathrm{eV}, \alpha_{0}=2.88 \mathrm{~cm}^{-1}$

\section{Spectral weights}

The measured spectral weight for the primary peck is determined by the parameter $\alpha_{0}$. With Eq. (60) we can converted that to $\sigma_{0}$. For $\mathrm{Sr}_{2} \mathrm{CuO}_{2} \mathrm{Cl}_{2}$ we have $\epsilon=5.5$ [22] and we get $\sigma_{0}=0.023 \Omega^{-1} \mathrm{~cm}^{-1}$ and for $\mathrm{La}_{2} \mathrm{CuO}_{4} \epsilon=$ 6.0 222] and $\sigma_{0}=0.018 \Omega^{-1} \mathrm{~cm}^{-1}$. We can use Eq. (58) to estimate $q_{\mathrm{A}}$. For $\mathrm{La}_{2} \mathrm{CuO}_{4}, V_{\mathrm{Cu}}=95 \times 10^{-24} \mathrm{~cm}^{3}$ and we approximate $M$ with an $\mathrm{O}$ mass (a reduced mass would be more appropriate). We get $q_{\mathrm{A}} / e=0.082$. For $\mathrm{Sr}_{2} \mathrm{CuO}_{2} \mathrm{Cl}_{2}$ with $V_{\mathrm{Cu}}=123 \times 10^{-24} \mathrm{~cm}^{3}$ we get $q_{\mathrm{A}} / e=$ 0.088. Examining the expression for the effective charge we find that is of order $q_{\mathrm{A}} / e \sim \frac{2 J U_{p d}}{\Delta^{2}} \sim 0.1$ in very good agreement with the experimental values. One should take into account however that the previous estimates neglect the weight in the side bands so that the observed spectral weight is in reality larger than the one estimated with $q_{\mathrm{A}} / e \sim 0.1$. This leaves room for the effects discussed in Sec. IVA.

\section{Prediction for $\mathrm{La}_{2} \mathrm{NiO}_{4}$}

In this case structural parameters are similar to the previous compound, $V_{\mathrm{Ni}}=94 \times 10^{-24} \mathrm{~cm}^{3}$ and $\epsilon_{1}=5.4$ however the ratio $J / \Delta$ is an order of magnitude smaller 
[31]. So we expect $q_{\mathrm{A}} \sim \frac{2 J U_{p d}}{\Delta^{2}} \sim 0.01$ which makes the absorption two orders of magnitude smaller than in the previous compounds. With this value of $q_{\mathrm{A}}$ we get $\sigma_{0}=3.7 \times 10^{-4} \Omega^{-1} \mathrm{~cm}^{-1}$. We have predicted the line shape taking the phonon frequency from neutron scattering experiments $\left(\omega_{\|}=0.066 \mathrm{eV}[28]\right)$ and the superexchange form Raman experiments $(J=0.030 \mathrm{eV}$ [31]). The expect absorption is plotted in Fig. 11. As explained before there are no sharp features in the $S=1$ case.

After this work has been completed we send the predicted line shape to J. Perkins and in response we received from them the experimental data plotted in Fig. 11. The agreement is remarkably good for the line shape and our rough estimation of the oscillator strength happened to be of the correct order of magnitude. Comparison with the previous case illustrates quite obviously the remarkably difference between the $S=1$ and the $S=1 / 2$ system the latter showing the strong side bands which require an explanation beyond RPA [27].

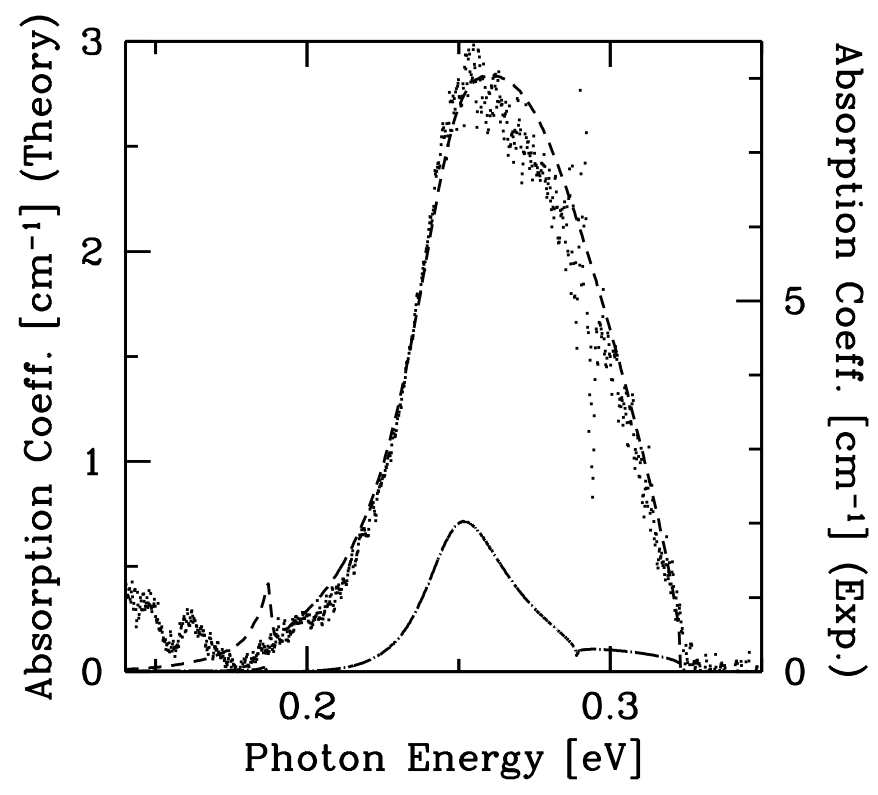

FIG. 11. Theoretical line shape for two-magnon absorption in $\mathrm{La}_{2} \mathrm{NiO}_{4}$ (dashed line). The dashed doted line is the contribution to the line shape from the bimagnon at $\boldsymbol{p}=(\pi, 0)$ Parameters are $\omega_{\|}=0.066 \mathrm{eV}$ 28], $J=0.030 \mathrm{eV}$, $\alpha_{0}=6.0 \times 10^{-2} \mathrm{~cm}^{-1}$. The dots are unpublish experimental data 29.

\section{DISCUSSION AND CONCLUSIONS}

The experiments [30] show also very strong side bands. These are expected as the effect of quantum fluctuations corresponding to the creation of an arbitrary even number of magnons. This is in principle also true for the Raman case and in fact a structure attributed to a four magnon process was observed in Raman [31] at a similar energy to the first side band (note that phonon energies are almost negligible here).

In the Ising limit one can roughly estimate the position of these side bands. The minimum energy to excite 4 magnons (plaquette configuration) is $\omega_{4 \mathrm{mag}}=4 J$ which correspond to the first side band (see Fig's. 9,10). The next side band is attributed to a process in which four spins are flipped in a column which has an excitation energy of $\omega_{4 \mathrm{mag}}=5 \mathrm{~J}$ in the Ising limit. In general one can show that side bands are expected at integer values of $J$ as observed experimentally. We have also computed the side bands by exact diagonalization of a small cluster [27]. The exact result confirms the Green function calculation and show the side bands corresponding to higher multimagnon process. The relative weight of the side bands seem to be smaller than in the experiments presumably because of finite site effects or the presence of other process in the magnetic Hamiltonian as in Raman [32, 33]. Exact diagonalization calculations of the Raman spectra [34] show also this kind of side bands.

As the temperature is raised one expects that the structure broadens in a way similar to the Raman line shape and at the same time a hot phonon band should appear at $E_{(\pi, 0)}^{\max }-\omega_{\|}$corresponding to a process in which a phonon of the bath is absorbed and a bimagnon is emitted. It would be interesting if this effect can be seen experimentally.

It is interesting to point out that one can in principle see the bimagnons for a particular momentum without the assistance of the phonon in neutron scattering experiments [35] or high resolution electron energy loss (HREELS) experiments. In the latter for energy losses small with respect to the energy of the incident electrons and in specular scattering geometry, dipole selection rules are valid and one should also see the analogue of the phonon-assisted IR line shape. Preliminary HREELS results [36] in $\mathrm{Sr}_{2} \mathrm{CuO}_{2} \mathrm{Cl}_{2}$ show a structure which agrees very well with Perkins et al. data. Other possibility to see this excitations without the assistance of the phonon would be to add impurities. In this way the zero total momentum selection rule will not apply any more and the narrow $(\pi, 0)$ bimagnon should give some signature at $E_{(\pi, 0)}^{\max }$. The impurity should also break inversion symmetry (which to same extent is always does) in order to make the two magnon excitations IR active and they should not introduce doping otherwise the doping dependent mid-IR band [37] would mask everything. Possible candidates would be $\mathrm{Nd}_{2-x} \operatorname{Pr}_{x} \mathrm{CuO}_{4}$ or similar combinations with other rare earths. Since the Nd is on top of a $\mathrm{Cu}$ the $\mathrm{O}$ is not any more in a center of inversion.

Another interesting experiment would be to repeat Perkins et al. experiment in an insulator with in-plane anisotropy. Since an electric field in the $x$ direction couples with bimagnons at $(\pi, 0)$ and one in the $y$ direction 
with bimagnons at $(0, \pi)$, if this two directions are not equivalent the primary peak should split in a twined crystal.

We do not believe that this mid-IR excitations are related to the doping dependent mid-IR bands observed in the cuprates for which a completely different mechanism has been proposed [38, 39. For example for $\mathrm{Nd}_{2} \mathrm{CuO}_{4-y}$ in the notation of Ref. 40 the band labeled $J$ at $0.16 \mathrm{eV}$ has been quite undoubtedly shown to be due to polaron formation 10,11,41,42 by Calvani et al. 443. The structure labeled $I$ at $0.76 \mathrm{eV}$ is the analogue of the mid-IR band reported by Uchida et al. [37] for $\mathrm{La}_{2-x} \mathrm{Sr}_{x} \mathrm{CuO}_{4}$ at $0.5 \mathrm{eV}$ and can be explained by a purely electronic mechanism 38, 39. Only the much weaker structure labeled $K$ at $0.34 \mathrm{eV}$ may be related to this excitations.

In this work we have computed effective coupling constants of light with multimagnon excitations assisted by phonons and the line shape of the primary peak. Our results explain recent measured absorption bands in the mid IR of parent cuprate superconductors and show that this technique proves high energy magnetic excitations. This is very interesting because IR spectroscopy is a technique intrinsically more accurate and with a much better signal to noise ratio than other techniques available like Raman or neutron scattering. We have demonstrated the existence of very sharp virtual bound states of magnons in spin $1 / 2$ systems at momentum $(\pi, 0)$ and show the shape of the excitations at different momentum. To the best of our knowledge is the first time that the RPA equations for the two magnon problem are presented for arbitrary total momentum.

\section{ACKNOWLEDGMENTS}

We acknowledge the authors of Ref. [4 for sending us their work previous to publication and for enlightening discussions and R. Eder and M. Meinders for helping us with the exact diagonalization calculations. This investigation was supported by the Netherlands Foundation for Fundamental Research on Matter (FOM) with financial support from the Netherlands Organization for the Advance of Pure Research (NWO) and Stichting Nationale Computer Faciliteiten (NCF). Computations where perform at SARA (Amsterdam). J.L. is supported by a postdoctoral fellowship granted by the Commission of the European Communities.

\section{APPENDIX A: VERTEX FUNCTIONS}

The symmetrized vertex in Eq. (34) are given by $\Gamma=$ $\Gamma^{\|}+\Gamma^{\perp}$ with

$$
\Gamma_{1234}^{\|}=-\frac{J z}{4}\left[\gamma_{2+1}\left(u_{1} v_{2} v_{3} u_{4}+v_{1} u_{2} u_{3} v_{4}\right)\right.
$$

$$
\begin{aligned}
& +\gamma_{2-4}\left(u_{1} u_{2} u_{3} u_{4}+v_{1} v_{2} v_{3} v_{4}+u_{1} v_{2} u_{3} v_{4}+v_{1} u_{2} v_{3} u_{4}\right) \\
& +1 \leftrightarrow 2+3 \leftrightarrow 4+1 \leftrightarrow 2 \text { and } 3 \leftrightarrow 4], \\
& \Gamma \frac{\perp}{1234}=-\frac{J z}{8}\left[\left(2 \gamma_{2}+\gamma_{3}\right) u_{1} u_{2} v_{3} u_{4}+\left(2 \gamma_{1}+\gamma_{3}\right) v_{1} v_{2} u_{3} v_{4}\right. \\
& +\left(2 \gamma_{3}+\gamma_{2}\right) u_{1} v_{2} u_{3} u_{4}+\left(2 \gamma_{4}+\gamma_{2}\right) v_{1} u_{2} v_{3} v_{4} \\
& +1 \leftrightarrow 2+3 \leftrightarrow 4+1 \leftrightarrow 2 \text { and } 3 \leftrightarrow 4] \text { ]. }
\end{aligned}
$$

In term of total momentum and relative momentum variables the vertex in Eq. (39) can be rewritten as

$$
\begin{aligned}
\Gamma_{\boldsymbol{p} \boldsymbol{q}_{1} \boldsymbol{q}_{2}}^{\|} & =-J\left[\sum_{\delta l=1,2}\left(f_{\boldsymbol{p} \boldsymbol{q}_{1}}^{l \delta} f_{\boldsymbol{p} \boldsymbol{q}_{2}}^{l \delta}+h_{\boldsymbol{p q}_{1}}^{l \delta} h_{\boldsymbol{p} \boldsymbol{q}_{2}}^{l \delta}\right)+\frac{z}{2} \gamma_{\boldsymbol{p}} f_{\boldsymbol{p} \boldsymbol{q}_{1}}^{3} f_{\boldsymbol{p} \boldsymbol{q}_{2}}^{3}\right] \\
\Gamma_{\boldsymbol{p} \boldsymbol{q}_{1} \boldsymbol{q}_{2}}^{\perp} & =-J \sum_{\delta}\left[\cos \left(\frac{p_{\delta}}{2}\right)\left(f_{\boldsymbol{p} \boldsymbol{q}_{1}}^{1 \delta} f_{\boldsymbol{p} \boldsymbol{q}_{2}}^{3}+\frac{1}{2} h_{\boldsymbol{p} \boldsymbol{q}_{1}}^{1 \delta} h_{\boldsymbol{p} \boldsymbol{q}_{2}}^{3}\right)\right. \\
& \left.+\frac{1}{2} \sin \left(\frac{p_{\delta}}{2}\right) h_{\boldsymbol{p} \boldsymbol{q}_{1}}^{2 \delta} h_{\boldsymbol{p} \boldsymbol{q}_{2}}^{4}+\boldsymbol{q}_{1} \leftrightarrow \boldsymbol{q}_{2}\right]
\end{aligned}
$$

where we used the definitions in Eqs. (37), (38).

\section{APPENDIX B: SOLUTION OF THE TWO MAGNON PROBLEM FOR 2D AND $p_{x}=p_{y}$}

In this case the Green functions for fixed $\boldsymbol{p}$ are invariant with respect to the exchange $q_{x} \leftrightarrow q_{y}$. As is done in the theory of Raman scattering we can define $G_{l, l^{\prime}}^{d}=2\left(G_{l x, l^{\prime} x}-G_{l x, l^{\prime} y}\right)$ and a similar definition for the noninteracting Green functions. In this case Eq. (45) reduce to a $2 \times 2$ problem,

$$
G_{l l^{\prime}}^{d}=G_{l l^{\prime}}^{d(0)}-\frac{J}{2} \sum_{l^{\prime \prime}=1,2} G_{l l^{\prime \prime}}^{d(0)} G_{l^{\prime \prime} l^{\prime}}^{d}
$$

which can be solved for

$G_{11}^{d}=$

$\frac{G_{11}^{d(0)}+\frac{J}{2}\left(G_{11}^{d(0)} G_{22}^{d(0)}-\left(G_{12}^{d(0)}\right)^{2}\right)}{1+\frac{J}{2}\left(G_{11}^{d(0)}+G_{22}^{d(0)}\right)+\frac{J^{2}}{4}\left(G_{11}^{d(0)} G_{22}^{d(0)}-\left(G_{12}^{d(0)}\right)^{2}\right)}$.

\section{APPENDIX C: SOLUTION OF THE TWO MAGNON PROBLEM FOR $\boldsymbol{p}=(\pi, 0,0 .$.}

In this case the Green function for the $x$ direction decouples from the rest in Eq. (45). This is because $G_{l x, 3}^{(0)}=0, G_{l x, l^{\prime} \delta}^{(0)}=0$ with $\delta \neq x$. To see this notice that in the definition Eq. (42), the kernel changes sign under the replacement $\boldsymbol{q} \rightarrow \boldsymbol{q}+(\pi, 0,0 .$.$) . So we get,$

$$
G_{l x l^{\prime} x}=G_{l x l^{\prime} x}^{(0)}-J \sum_{l^{\prime \prime}=1,2} G_{l x l^{\prime \prime} x}^{(0)} G_{l^{\prime \prime} x l^{\prime} x}
$$

we can solve for 
$G_{1 x 1 x(\pi, 0,0 \ldots)}=$

$\frac{G_{1 x 1 x}^{(0)}+G_{2 x 2 x}^{(0)}+2 G_{1 x 2 x}^{(0)}+4 J\left(G_{1 x 1 x}^{(0)} G_{2 x 2 x}^{(0)}-\left(G_{1 x 2 x}^{(0)}\right)^{2}\right)}{1+2 J\left(G_{1 x 1 x}^{(0)}+G_{2 x 2 x}^{(0)}\right)+4 J^{2}\left(G_{1 x 1 x}^{(0)} G_{2 x 2 x}^{(0)}-\left(G_{1 x 2 x}^{(0)}\right)^{2}\right)}$.

\section{APPENDIX D: HIGH ENERGY APPROXIMATION}

When the line shape is positioned at sufficiently high energies $\left(\omega>E_{\mathrm{m}}\right)$ it is a good approximation to neglect the $v$ 's respect to the $u$ 's in Eqs. (36a-38). In principle this can be done everywhere in the expressions for the Green functions. However it is convenient to do this in two steps. First we define

$$
f_{\boldsymbol{p q}}^{ \pm \delta}=f_{\boldsymbol{p q}}^{1 \delta} \pm f_{\boldsymbol{p q}}^{2 \delta}
$$

and we do the HE approximation in the vertex, i.e. we neglect all contributions that involve $v$ 's in Eq. (A3). We denote with primes the quantities computed in the HE approximation, i.e.

$$
\begin{aligned}
& \Gamma_{\boldsymbol{p} \boldsymbol{q}_{1} \boldsymbol{q}_{2}}^{\prime}=-2 J \sum_{\delta} f_{\boldsymbol{p} \boldsymbol{q}_{1}}^{+\delta} f_{\boldsymbol{p} \boldsymbol{q}_{2}}^{+\delta} \\
& \Gamma_{\boldsymbol{p q}_{1} \boldsymbol{q}_{2}}^{\prime \perp}=0
\end{aligned}
$$

Now we rederive Eq. (45) in the HE approximation.

$$
G_{\mu \nu}^{\prime}=G_{\mu \nu}^{(0)}-2 J \sum_{\delta} G_{\mu,+\delta}^{(0)} G_{+\delta, \nu}^{\prime} .
$$

We can treat $\delta$ as a matrix or vector index of dimension $\mathrm{d}$ and put this as

$$
G_{l l^{\prime}}^{\prime}=G_{l l^{\prime}}^{(0)}-2 J G_{l+}^{(0)} G_{+l^{\prime}}^{\prime} .
$$

Notice that $G_{11}^{\prime}, G_{22}^{\prime}, G_{ \pm \pm}^{\prime}$ are matrixes $G_{13}^{\prime}, G_{31}^{\prime}$ are vectors and $G_{33}^{\prime}$ is a scalar. Solving the $\mathrm{d} \times \mathrm{d}$ problem for $G_{+l}^{\prime}$ we get

$$
G_{+l}^{\prime}=\left(1+2 J G_{++}^{(0)}\right)^{-1} G_{+l}^{(0)} .
$$

and replacing in Eq. (D4) we get also $G_{l l^{\prime}}^{\prime}$. We also have the exact transformation

$$
\begin{aligned}
G_{11}^{\prime} & =\frac{1}{4}\left(G_{++}^{\prime}+G_{--}^{\prime}+2 G_{+-}^{\prime}\right), \\
G_{31}^{\prime} & =\frac{1}{2}\left(G_{3+}^{\prime}+G_{3-}^{\prime}\right), \\
G_{13}^{\prime} & =\frac{1}{2}\left(G_{+3}^{\prime}+G_{-3}^{\prime}\right) .
\end{aligned}
$$

When calculating the Green function for the two magnon operator, Eq. (46) one would be tempted to make the same approximation and keep only $G_{++}^{\prime}$ since according to the definitions Eqs. (36), (37), (43), all other contributions are negligible. This means that not only the vertex or equivalently the interaction part of the Hamiltonian $\left(V_{\text {res }}^{\mathrm{RPA}}\right)$ is approximated by $\left(V_{\mathrm{res}}^{\prime} \mathrm{RPA}\right)$ but also the operator $\delta B_{p}^{x}$ is approximated by $\delta B_{p}^{\prime x}$. We call this the HE approximation in the vertex and in the operator. Although this is perfectly consistent at high energies this approach produces a large spurious contribution at low energies. To see this consider the case of $\boldsymbol{p}=0$. We can define

$$
\begin{aligned}
& \delta B_{0}^{s}=\delta B_{0}^{x}+\delta B_{0}^{y}, \\
& \delta B_{0}^{d}=\delta B_{0}^{x}-\delta B_{0}^{y}
\end{aligned}
$$

Now we find,

$$
\begin{aligned}
\left\langle\left\langle\delta B_{0}^{x} ; \delta B_{0}^{x}\right\rangle\right\rangle=\frac{1}{4} & \left(\left\langle\left\langle\delta B_{0}^{d} ; \delta B_{0}^{d}\right\rangle\right\rangle+\left\langle\left\langle\delta B_{0}^{s} ; \delta B_{0}^{d}\right\rangle\right\rangle\right. \\
& \left.+\left\langle\left\langle\delta B_{0}^{d} ; \delta B_{0}^{s}\right\rangle\right\rangle+\left\langle\left\langle\delta B_{0}^{s} ; \delta B_{0}^{s}\right\rangle\right\rangle\right) .
\end{aligned}
$$

Only the first term contributes to the line shape. i.e the $\boldsymbol{p}=0$ contribution to the IR line shape is identical to the Raman line shape. This follows from the fact that $B_{0}^{s}$ is proportional to the Hamiltonian and hence it commutes with it. To enforce that in spin-wave theory, we would like $B_{0}^{s}$ to commute with $H$ order by order in $1 / S$. However if we do the high energy approximation in the operator of Eq. (46), we miss some terms of order $S$ in $B_{0}^{s}$ and then we find that to order $S^{2},\left[\delta B_{p}^{\prime s}, H_{0}\right] \neq 0$. This gives spurious scattering in the $s$ channel. We can avoid that by doing the high energy approximation in the vertex alone. In this case $G_{13}^{\prime}, G_{31}^{\prime}$ and $G_{33}^{\prime}$ are kept in Eq. (46) and computed with Eqs. (D4), (D5) and (D6). It is instructive to check analytically that in this way the non-interacting $\left\langle\left\langle\delta B_{0}^{s} ; \delta B_{0}^{s}\right\rangle\right\rangle^{0}=0$.

In the 2 -d case we find,

$$
\left(1+2 J G_{++}^{(0)}\right)^{-1}=\frac{1}{\Delta_{++}}\left(\begin{array}{cc}
1+2 J G_{y+y+}^{(0)} & -2 J G_{x+y+}^{(0)} \\
-2 J G_{x+y+}^{(0)} & 1+2 J G_{x+x+}^{(0)}
\end{array}\right)
$$

with

$$
\begin{aligned}
\Delta_{++}=1 & +2 J\left(G_{x+x+}^{(0)}+G_{y+y+}^{(0)}\right) \\
& +4 J^{2}\left[G_{x+x+}^{(0)} G_{y+y+}^{(0)}-\left(G_{x+y+}^{(0)}\right)^{2}\right] .
\end{aligned}
$$

so we get for $G_{+x+x}^{\prime}$

$G_{+x+x}^{\prime}=\frac{G_{+x+x}^{(0)}+2 J\left[G_{+x+x}^{(0)} G_{+y+y}^{(0)}-\left(G_{+x+y}^{(0)}\right)^{2}\right]}{\Delta_{++}}$

This reduce to Eq. (55) for $\boldsymbol{p}=(\pi, 0)$ using the properties of Appendix Q. 
* Present Address: Lab. Léon Brillouin, CE de Saclay, 91191 Gif-sur-Yvette, CEDEX, France.

[1] R. Newman and R. M. Chrenko, Phys. Rev. 114, 1507 (1959).

[2] Y. Tanabe, T. Moriya, and S. Suagano, Phys. Rev. Lett. 15, 1023 (1965).

[3] Y. Mizuno and S. Koide, Phys. Kondens. Materie 2, 179 (1964).

[4] J. D. Perkins et al., Phys. Rev. Lett. 71, 1621 (1993).

[5] J. D. Perkins, Ph.D. thesis, Massachusetts Institute of Technology, Cambridge, Massachusetts, 1994.

[6] H. Eskes, L. H. Tjeng, and G. A. Sawatzky, Phys. Rev. B 42, 288 (1990).

[7] A. K. McMahan, J. F. Annett, and R. M. Martin, Phys. Rev. B 42, 6268 (1990).

[8] E. Manousakis, Rev. Mod. Phys. 63, 1 (1991).

[9] J. Lorenzana and G. A. Sawatzky, Phys. Rev. Lett. 74, 1867 (1995). (supr-con/9501001)

[10] K. Yonemitsu, A. Bishop, and J. Lorenzana, Phys. Rev. Lett. 69, 965 (1992).

[11] K. Yonemitsu, A. Bishop, and J. Lorenzana, Phys. Rev. B 47, 12059 (1993).

[12] J. C. Slater and G. F. Koster, Phys. Rev. B 94, 1498 (1954).

[13] H. Eskes and J. H. Jefferson, Phys. Rev. B 48, 9788 (1993).

[14] J. Zaanen and G. A. Sawatzky, Can. J. Phys. 65, 1262 (1987).

[15] M. J. Rice, Solid State Commun. 31, 93 (1979).

[16] D. N. Zubarev, Sov. Phys. Uspk. 3, 320 (1960), [Usp. Fiz. Nauk 71, 71 (1960)].

[17] C. M. Canali and S. M. Girvin, Phys. Rev. B 45, 7127 (1992).

[18] J. B. Parkinson, J. Phys. C (Solid St. Phys.) 2, 2012 (1969).

[19] R. J. Elliott and M. F. Thorpe, J. Phys. C (Solid St. Phys.) 2, 1630 (1969).

[20] R. J. Elliott et al., Phys. Rev. Lett. 21, 147 (1968).

[21] H. Rietschel, L. Pintschovious, and W. Reichardt, Physica C 162-164, 1705 (1989).

[22] S. Tajima et al., Phys. Rev. B 43, 10496 (1991).

[23] R. R. P. Singh, P. A. Fleury, K. B. Lyons, and P. E. Sulewsky, Phys. Rev. Lett. 62, 2736 (1989).

[24] Y. Tokura et al., Phys. Rev. B 41, 11657 (1990).

[25] J. D. Perkins and J. M. Graybeal, personal com. (unpublished).

[26] This is analogous to what was found for $\mathrm{FeF}_{2}$ see $\mathrm{T}$. Moriya, J. Phys. Soc. Jpn. 21, 926 (1966).

[27] J. Lorenzana, R. Eder, M. Meinders, and G. A. Sawatzky, J. of Superconductivity 8, 567 (1995), to be published.

[28] L. Pintschovious et al., Phys. Rev. B 40, 3770 (1989).

[29] J. D. Perkins, D.S. Kleinberg, M. A. Kastner , R. J. Birgeneau, Y. Endoh, K. Yamada and S. Hosoya, Infrared Optical Excitations in $\mathrm{La}_{2} \mathrm{NiO}_{4}$. Preprint.

[30] J. P. Falck, A. Levy, M. A. Kastner, and R. J. Birgeneau, Phys. Rev. B 48, 4043 (1993).
[31] S. Sugai, Phys. Rev. B 42, 1045 (1990).

[32] M. Roger and J. M. Delrieu, Phys. Rev. B 39, 2299 (1989).

[33] Y. Honda, Y. Kuramoto, and T. Watanabe, Physica C 185-189, 1493 (1991).

[34] E. Gagliano and S. Bacci, Phys. Rev. B 42, 8772 (1990).

[35] R. A. C. W. J. L. Buyers, P. Martel, and R. W. H. Stevenson, Phys. Rev. Lett. 23, 86 (1969).

[36] J. J. M. Pothuizen and G. A. Sawatzky (unpublished).

[37] S. Uchida et al., Phys. Rev. B 43, 7942 (1991).

[38] J. Lorenzana and L. Yu, Phys. Rev. Lett. 70, 861 (1993).

[39] J. Lorenzana, to be published in Physica B.

[40] G. A. Thomas et al., Phys. Rev. Lett. 67, 2906 (1991).

[41] A. Dobry, A. Greco, J. Lorenzana, and J. Riera, Phys. Rev. B 49, 505 (1994).

[42] J. Lorenzana and A. Dobry, Phys. Rev. B 50, 16094 (1994).

[43] P. Calvani et al., Solid State Commun. 91, 113 (1994). 